\title{
Alternate Event-triggered Aperiodically Intermittent Control for Synchronization of Multi-weighted Complex Networks
}

\section{Dongsheng Xu}

Harbin Institute of Technology Weihai

Huan Su ( $\square$ suhuantg@hitwh.edu.cn )

Harbin Institute of Technology Weihai https://orcid.org/0000-0001-8057-6664

Chenfei Guo

Harbin Institute of Technology Weihai

\section{Research Article}

Keywords: multi-weighted complex networks, alternate event-triggered control, aperiodically intermittent control, exponential synchronization

Posted Date: March 2nd, 2021

DOI: https://doi.org/10.21203/rs.3.rs-216783/v1

License: (9) This work is licensed under a Creative Commons Attribution 4.0 International License. Read Full License 


\title{
Alternate Event-triggered Aperiodically Intermittent Control for Synchronization of Multi-weighted Complex Networks
}

\author{
Dongsheng $\mathrm{Xu}$, Chenfei Guo and Huan $\mathrm{Su}^{*}$
}

\begin{abstract}
In this paper, the exponential synchronization problem for multi-weighted complex networks via alternate eventtriggered aperiodically intermittent control (AETAIC) is considered. Different from existing literature, the proposed AETAIC is triggered alternatively by two pre-defined conditions, which can fast react to asynchronous external events and show better real-time control performance. Meanwhile, AETAIC removes the restrictions of traditional intermittent control on the lower bound of control intervals and upper bound of control periods or the maximum proportion of rest intervals. Though graph theory and Lyapunov method, several sufficient conditions are given to ensure exponential synchronization of the studied networks and Zeno behaviors can be excluded. Moreover, the theoretical results demonstrate that the control gain affects the control widths and exponential convergence rate, which shows that AETAIC can further reduce the frequency of controller updates and release the computation burdens. Finally, in order to illustrate the theoretical results, two practical applications about Chua's circuits and coupled oscillators are presented. Meanwhile, numerical simulations are provided to validate the effectiveness of the results.
\end{abstract}

Index Terms-multi-weighted complex networks, alternate event-triggered control, aperiodically intermittent control, exponential synchronization

\section{INTRODUCTION}

Over the past few years, due to their extensive application in the social, economic, and biological fields, researches on complex networks have increased, and many interesting results have been obtained [1]- [3]. Generally, complex networks consist of a set of interconnected nodes, and each node can be described as a unit of the system in the real life. However, with the development of network science, single-weighted complex networks cannot easily describe many real-world networks. In transportation networks, for example, people always choose multiple modes of travel, such as airplanes, high-speed rail, and cars, which results in multi-weighted complex networks (MWCNs). Different from single-weighted complex networks, multi-weighted complex networks contain more complicated dynamic behaviors and performances due to their complicated

Dongsheng $\mathrm{Xu}$ and Huan $\mathrm{Su}$ are with the Department of Mathematics, Chenfei Guo is with School of Materials Science and Engineering, Harbin Institute of Technology, Weihai, 264209, China. (e-mail: xudongsheng555@163.com,gcfphoenix@126.com, suhuantg@hitwh.edu.cn).

This work was supported by the Shandong Province Natural Science Foundation (Nos. ZR2018MA005, ZR2018MA020, ZR2017MA008); the Innovation Technology Funding Project in Harbin Institute of Technology (No. HIT.NSRIF.201703) and the Project of Shandong Province Higher Educational Science and Technology Program of China (No. J18KA218).

Corresponding author: Huan $\mathrm{Su}$. topological structures, and there are more than one connection between a pair of nodes with their distinct properties. Thereby, MWCNs can simulate more complicated networks like social networks, transportation networks, neural networks and so forth [4]- [7]. As one typical dynamical behavior of MWCNs, synchronization has attracted much attention from scholars and intensive research has been carried out [7]- [12]. In [7], the output synchronization problems for MWCNs are discussed by employing the pinning control. In [9], authors investigate the pinning synchronization of directed and undirected complex dynamical networks with multiple weights. On the basis of graph theory, the synchronization for stochastic MWCNs with Lévy noise is realized in [10].

Nevertheless, in many cases, MWCNs fail to realize synchronization spontaneously due to the complexity of topological structure and node dynamics. Thus, various control strategies, including continuous control and discontinuous control [13]- [19] are developed to achieve synchronization of MWCNs. However, continuous control requires long-lasting communication consumption and information measurement which encounters infeasibility in limited resources. Thus, studies have concentrated on discontinuous ones, especially intermittent control [20]- [23]. It is worth mentioning that intermittent control mentioned above is periodic, with fixed control periods and control bandwidths. However, in some natural phenomena such as the generation of the wind power with small grid, the results remain conservative. Therefore, aperiodically intermittent control (AIC) which can reduce cost$\mathrm{s}$ and communication burdens is generalized to handle more intricate cases [24]- [26]. In the aforementioned literatures, restrictions on the lower bound of control intervals and upper bound of control periods or the maximum proportion of rest intervals are unavoidable assumptions. The description of AIC strategy is shown in Figure 1 for better understanding of the constraint. In detail, define $\left(t_{i}, s_{i}\right]$ as the control interval and $\left[s_{i}, t_{i+1}\right)$ as the rest interval, assumptions in the following are utilized:

$$
\begin{aligned}
& \text { (1) } \inf _{i \in N}\left(s_{i}-t_{i}\right)=\theta, \sup _{i \in N}\left(t_{i+1}-t_{i}\right)=\omega, 0<\theta<\omega<+\infty, \\
& \text { (2) } \limsup _{i \rightarrow \infty} \frac{t_{i+1}-s_{i}}{t_{i+1}-t_{i}}=\Psi, \Psi \in(0,1) .
\end{aligned}
$$

It can be seen that although the intermittent control is set aperiodically, control and rest intervals are uniformly distributed which is very conservative and not easy to implement in real applications. Thus, traditional AIC fails to obtain complete 


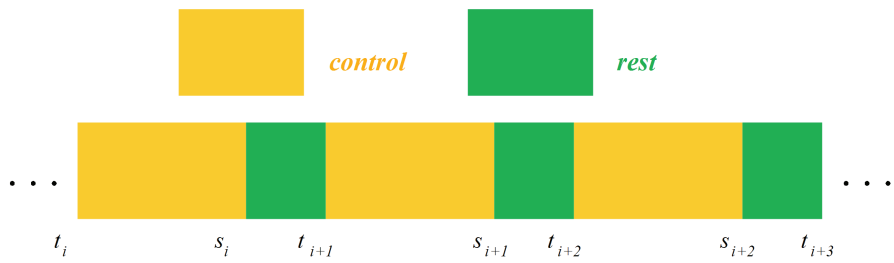

Fig. 1: Sketch map of AIC strategy

aperiodicity and its flexibility are undoubtedly undermined. Moreover, the actuation of AIC depends on those triggering time instants $t_{i}$ and $s_{i}$, meaning that the actuation is irrelevant to the dynamical evolution of MWCNs. In that case, control will be unnecessarily triggered and yet it may fail to show the real-time reaction to asynchronous external events, which gives rise to the poor efficiency of time-triggered AIC.

In an effort to overcome the constraints brought by timetriggered control, event-triggered control (ETC) is introduced to avoid useless control actuation and improve the reaction ability of AIC, where controller is updated only when a certain event is triggered. The triggering conditions of ETC is related to the current state, rather than time instants [27]- [32]. In [31], ETC is employed to obtain leader-following consensus of multi-agent systems. Exponential anti-synchronization problem is coped with under ETC in [32]. It is mentionable that control strategies adopted by those literatures are considered to be single-event triggered, where controller will be operating continuously once it is actuated. To avoid this situation, a novel type of control-alternate event-triggered aperiodically intermittent control (AETAIC), which is activated and off by two alternative trigger conditions, is proposed. The AETIC proposed in this paper avoids continuous work such as ETC, and the time span of controller's operation is significantly shortened, so the update frequency can be further reduced. To the best of our knowledge, there is no result reported on the synchronization of MWCNs by using AETAIC, so it is still of great research significance.

Motivated by the above-mentioned discussion, the synchronization problem for MWCNs via AETAIC is considered in this paper. In particular, AETAIC is the improvement of the traditional ETC and AIC. Several sufficient conditions for the synchronization are acquired by employing graph theory and Lyapunov method. In this case, Zeno behaviors can be excluded. Finally, applications about Chua's circuits and coupled oscillators are presented with corresponding numerical simulations to validate the feasibility of theoretical results. Based on the proposed AETAIC, we investigate the exponential synchronization of MWCNs, which is an interesting topic worth studying. The main contribution of this paper can be synthesized as follows

- It is noteworthy that to achieve synchronization, we consider that at least one directed path from one to another are supposed to exist for different vertices in the digraph instead of all sub-digraphs. Thus, the requirement of network topological structure can be reduced, which shows more practical significance in real life.

- A new type of control AETAIC is introduced to solve synchronization problems for MWCNs. On the basis of AETAIC, assumptions on the minimum/maximum width of control intervals or the minimum activation time ratio of AIC in [24]- [26] are removed to realize more complete aperiodicity of AIC.

- Chua's circuits and coupled oscillators are intensively investigated due to their wide applications nowadays. In this paper, AETAIC is utilized to solve the synchronization problems for both two models. Different from traditional ETC [27]- [30], the triggering time instants in AETAIC not only define the update of controller, but also determines its startup and shutdown. Thereby, continuous operation of controller can be avoided and update frequency can be reduced.

The rest of this paper is organized as follows. In Section II, several preliminaries and models are described in detail. The main results will be developed in Section III. Two applications and the corresponding numerical simulations are provided in Section IV.

\section{PRELIMINARIES AND MOdel DESCRIPTIONS}

2.1 Notations. First of all, let $\mathbb{R}$ denote the set of real numbers, $\mathbb{R}^{+}$denote the set of nonnegative real numbers, $\mathbb{R}^{n}$ denote the $n$-dimensional Euclidean space and $\mathbb{R}^{n \times m}$ denote the set of all $n \times m$ real matrices. In addition, " $\mathrm{T}$ " is defined as the transpose of a vector or a matrix. Let $\mathcal{N}=\{1,2, \cdots, N\}$ and $\mathcal{L}=\{1,2, \cdots, L\}$. Moreover, $|\cdot|$ is defined as the Euclidean norm of a matrix or a vector.

In terms of the graph theory, some basic concepts will be given in the following. A digraph $\mathcal{K}(\mathcal{N}, \varepsilon)$ is defined by a set $\mathcal{N}$ of vertices and a set $\varepsilon$ of $\operatorname{arcs}(k, h)$ from vertex $k$ to vertex $h$. $\operatorname{Graph} \mathcal{K}(\mathcal{N}, \varepsilon)$ is supposed to be strongly connected if there exists a path that follows the direction of the edges between any pair of different nodes. Moreover, digraph $(\mathcal{K}, \mathbb{B})$ denotes a digraph $\mathcal{K}$ with single weight matrix $\mathbb{B}=\left(b_{k h}\right)_{N \times N}$, where $b_{k h}>0$ stands for the weight of arc $(h, k)$ if it exists, and $b_{k h}=0$ otherwise. The Laplacian matrix of $(\mathcal{K}, \mathbb{B})$ is defined as $L(\mathcal{K}, \mathbb{B})=\left(l_{k h}\right)_{N \times N}$, where $l_{k h}=-b_{k h}$ for $h \neq k$ and $l_{k h}=\sum_{i \neq k} b_{k i}$ for $k=h(k, h \in \mathcal{N})$. Since the graph $\mathcal{K}$ has multiple weight matrices $\mathbb{B}_{s}$, where $s \in \mathcal{L}$, we can also describe the graph as $\left(\mathcal{K}, \mathbb{B}_{1}, \mathbb{B}_{2}, \cdots, \mathbb{B}_{L}\right)$ and separate it into several graphs with same nodes $\left(\mathcal{K}, \mathbb{B}_{1}\right),\left(\mathcal{K}, \mathbb{B}_{2}\right), \cdots,\left(\mathcal{K}, \mathbb{B}_{L}\right)$. More information can be found out in [37].

2.2 Model description. In this section, consider the following MWNCs built on a digraph $\left(\mathcal{K}, \mathbb{B}_{1}, \mathbb{B}_{2}, \cdots, \mathbb{B}_{L}\right)$

$$
\mathrm{d} x_{k}(t)=\left[f_{k}\left(x_{k}(t), t\right)+\sum_{l=1}^{L} \sum_{h=1}^{N} b_{k h}^{l}(t) H_{k h l}\left(x_{k}(t), x_{h}(t)\right)\right] \mathrm{d} t
$$

where $x_{k}(t)=\left(x_{k 1}(t), x_{k 2}(t), \cdots, x_{k m}(t)\right)^{\mathrm{T}} \in \mathbb{R}^{m}$ represents the state vector of the $k$ th node, $f_{k}(\cdot): \mathbb{R}^{m} \times \mathbb{R}^{+} \rightarrow \mathbb{R}^{m}$ is a continuous vector function, $H_{k h l}(\cdot, \cdot): \mathbb{R}^{m} \times \mathbb{R}^{m} \rightarrow \mathbb{R}^{m}$ 
stands for a coupling function. Meanwhile, $b_{k h}^{l}(t)$ are the weights of the edges between node $k$ and node $h$.

Throughout this paper, consider system (1) as the drive system, then the corresponding response system can be given by

$$
\begin{aligned}
\mathrm{d} y_{k}(t)= & {\left[f_{k}\left(y_{k}(t), t\right)+\sum_{l=1}^{L} \sum_{h=1}^{N} b_{k h}^{l}(t) H_{k h l}\left(y_{k}(t), y_{h}(t)\right)\right.} \\
& \left.+u_{k}(t)\right] \mathrm{d} t, k \in \mathcal{N}
\end{aligned}
$$

where $y_{k}(t)=\left(y_{k 1}(t), y_{k 2}(t), \cdots, y_{k m}(t)\right)^{\mathrm{T}} \in \mathbb{R}^{m}$ and $u_{k}(t)$ is AETAIC which can be designed as

$$
u_{k}(t)=\left\{\begin{array}{cl}
-a_{k}\left(y_{k}\left(t_{s}\right)-x_{k}\left(t_{s}\right)\right), & t \in\left[t_{s}, t_{s+1}^{\prime}\right), \\
0, & t \in\left[t_{s+1}^{\prime}, t_{s+1}\right),
\end{array}\right.
$$

where $a_{k}$ denotes the control gain. The span $\left[t_{s}, t_{s+1}^{\prime}\right)$ and $\left[t_{s+1}^{\prime}, t_{s+1}\right)$ are control interval and rest interval, respectively, in which $k=0,1,2, \ldots$. Meanwhile, $t_{s+1}^{\prime}-t_{s}$ denotes the $(s+1)$ th control width, $t_{s+1}-t_{s+1}^{\prime}$ denotes the $(s+1)$ th rest width. Moreover, we suppose $t_{0}=0$. Besides, the event-triggered control instant $t_{s}$ and rest instant $t_{s+1}^{\prime}$ are determined respectively by the following event-triggered conditions:

$$
\begin{aligned}
& t_{s+1}=\inf \left\{t>t_{s+1}^{\prime}:|N(t)|^{2}-\delta_{1}\left|e\left(t_{s+1}^{\prime}\right)\right|^{2} e^{\alpha\left(t-t_{s+1}^{\prime}\right)}>0\right\}, \\
& t_{s+1}^{\prime}=\inf \left\{t>t_{s}:|E(t)|^{2}-\delta_{2}\left|e\left(t_{s}\right)\right|^{2} e^{-\beta\left(t-t_{s}\right)}>0\right\},
\end{aligned}
$$

where $\delta_{1}, \delta_{2}, \alpha, \beta$ are positive constants, $N(t)=e(t)-e\left(t_{s+1}^{\prime}\right)$ and $E(t)=e(t)-e\left(t_{s}\right)$ represent the measurement errors.

Remark 1: In this paper, AETAIC (3) with event-triggered conditions (4) is designed. It can be seen that when the control interval $\left(t_{s}, t_{s+1}^{\prime}\right]$ becomes zero, then $u_{k}(t)$ will degenerate into event-triggered impulsive control [33]- [36], [41]. If rest interval $\left[t_{s+1}^{\prime}, t_{s+1}\right)$ turns into zero, then $u_{k}(t)$ will be continuous. Additionally, when the control and rest intervals are fixed, AETAIC will degenerate into periodically intermittent control triggered by time instants. Compared with those control strategies, it is apparent that AETAIC is more economical and shows less conservativeness, with more complete aperiodicity and flexibility.

Remark 2: Different from general ETC [27]- [30], in this paper, we introduce a novel type of control- AETAIC into MWCNs. For general ETC, the system is required to keep transmitting the information of state at sampling time instants until the next update of the controller. In other words, the controller will keep operating once it is actuated. In contrast to that, in AETAIC, two alternative triggering conditions are given respectively, on the basis of which triggering time instants not only determines the update of controller, but also determines its actuation and off. Thus, the frequency of controller updates in AETAIC are greatly reduced and unnecessary update of information can be avoided in order to save cost.
Then, let $e_{k}(t)=y_{k}(t)-x_{k}(t)$ be the synchronization error, and the error system can be described as

$$
\begin{aligned}
\mathrm{d} e_{k}(t)= & {\left[f_{k}\left(e_{k}(t), t\right)+\sum_{l=1}^{L} \sum_{h=1}^{N} b_{k h}^{l}(t) H_{k h l}\left(e_{k}(t), e_{h}(t)\right)\right.} \\
& \left.+u_{k}(t)\right] \mathrm{d} t, k \in \mathcal{N} .
\end{aligned}
$$

in which $f_{k}\left(e_{k}(t), t\right)=f_{k}\left(y_{k}(t), t\right)-f_{k}\left(x_{k}(t), t\right)$, $H_{k h l}\left(e_{k}(t), e_{h}(t)\right)=H_{k h l}\left(y_{k}(t), y_{h}(t)\right)-H_{k h l}\left(x_{k}(t), x_{h}(t)\right)$.

In the following, a definition and some assumptions given below will be used in our main results.

Definition 2.1: Drive system (1) and response system (2) achieve the exponential synchronization in mean square, if there exist constants $\varpi>0$ and $M>0$, such that

$$
|y(t)-x(t)|^{2} \leq M e^{-\varpi t}, t \geq 0,
$$

in which $x(t)=\left(x_{1}^{T}(t), x_{2}^{T}(t), \ldots, x_{N}^{T}(t)\right)^{T} \in \mathbb{R}^{m N}$ and $y(t)=\left(y_{1}^{T}(t), y_{2}^{T}(t), \ldots, y_{N}^{T}(t)\right)^{T} \in \mathbb{R}^{m N}, \varpi$ is called the convergence rate of exponential synchronization.

Assumption 2.1: For $k \in \mathcal{N}$, there exists a positive constant $\xi_{k}$ such that

$$
\left(y_{k}-x_{k}\right)^{\mathrm{T}}\left(f_{k}\left(y_{k}, t\right)-f_{k}\left(x_{k}, t\right)\right) \leq \xi_{k}\left|y_{k}-x_{k}\right|^{2},
$$

for any $x_{k}, y_{k} \in \mathbb{R}^{m}$.

Assumption 2.2: For $k, h \in \mathcal{N}, l \in \mathcal{L}$, there exist positive constants $\rho_{k}^{l}, \sigma_{k}^{l}$ such that

$$
\left|H_{k h l}\left(y_{k}, y_{h}\right)-H_{k h l}\left(x_{k}, x_{h}\right)\right| \leq \rho_{k}^{l}\left|y_{k}-x_{k}\right|+\sigma_{k}^{l}\left|y_{h}-x_{h}\right|,
$$

for any $x_{k}, x_{h}, y_{k}, y_{h} \in \mathbb{R}^{m}$.

Assumption 2.3: Assume that digraph $(\mathcal{K}, \mathbb{B})$ is strongly connected, where $\mathbb{B}=\left(\tilde{b}_{k h}\right)_{N \times N}, \tilde{b}_{k h}=\max _{t \geq 0, l \in \mathcal{L}}\left\{b_{k h}^{l}(t)\right\}$

Assumption 2.4: For all $s=0,1,2, \ldots$, there exists positive constants $\varepsilon$ and $T_{s}$, such that the following inequality holds

$$
\int_{t}^{t+T_{s}} \tilde{B}(\tau) \mathrm{d} \tau \leq \varepsilon
$$

where $\tilde{B}(t)=\max _{\substack{k, h \in \mathcal{N} \\ l \in \mathcal{L}}}\left\{b_{k h}^{l}(t)\right\}$ and $T_{s} \in\left(0, t_{s+1}^{\prime}-t_{s}\right)$.

Remark 3: Figure. 2 depicts a digraph $\mathcal{K}$ composed of five vertices and its four sub-diagraphs. Under Assumption 3, we require that the digraph $(\mathcal{K}, \mathbb{B})$ is strongly connected. However, the sub-digraphs $\left(\mathcal{K}, \mathbb{B}_{1}\right),\left(\mathcal{K}, \mathbb{B}_{2}\right),\left(\mathcal{K}, \mathbb{B}_{3}\right),\left(\mathcal{K}, \mathbb{B}_{4}\right)$ do not need to be strongly connected. To be more specific, at least one directed path from one to another are supposed to exist for any pair of different vertices in diagraph $\mathcal{K}$ instead of all subdigraphs $\left(\mathcal{K}, \mathbb{B}_{1}\right),\left(\mathcal{K}, \mathbb{B}_{2}\right),\left(\mathcal{K}, \mathbb{B}_{3}\right)$. Thus, the requirements of network topological structures can be reduced, which shows more practical significance in real life.

\section{MAIN RESUltS}

In this section, the exponential synchronization of driveresponse systems (1) and (2) under AETAIC (3) is investigated. Before giving the main results, two lemmas are given as 


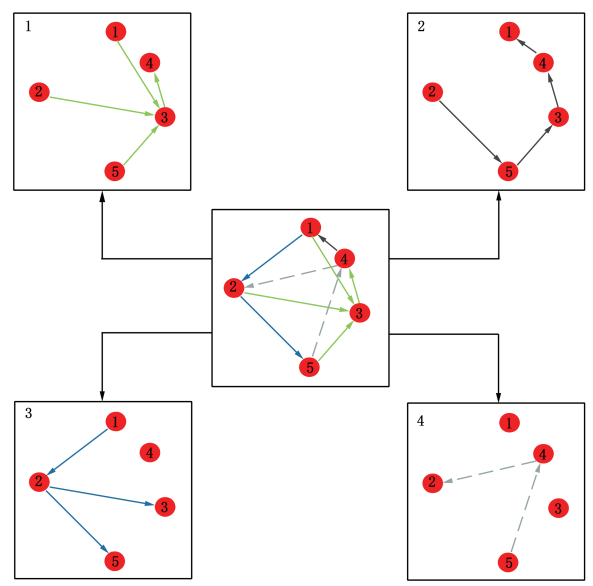

Fig. 2: Digraph $(\mathcal{K}, \mathbb{B})$ and its four sub-digraphs $\left(\mathcal{K}, \mathbb{B}_{1}\right)$, $\left(\mathcal{K}, \mathbb{B}_{2}\right),\left(\mathcal{K}, \mathbb{B}_{3}\right)$ and $\left(\mathcal{K}, \mathbb{B}_{4}\right)$

follows.

$$
\begin{aligned}
& \varphi_{1}(t)=\max _{k \in \mathcal{N}}\left(2 \xi_{k}-2 a_{k}+L N\left(2 \tilde{\rho}_{k}+\tilde{\sigma}_{k}\right) \tilde{B}(t)+L \sum_{h=1}^{N} Q_{k h}\right. \\
& \left.+2 \lambda_{1}\right), \varphi_{2}=\max _{k \in \mathcal{N}}\left\{\frac{a_{k}^{2} \delta_{2} \bar{d}}{2 \lambda_{1} \underline{d}}\right\}, \varphi_{3}=e^{\ell}, \quad \varphi_{4}=\frac{\varphi_{2} \varphi_{3}}{\beta}, \\
& \varphi_{5}=\varphi_{4} V\left(e\left(t_{s}\right), t_{s}\right), \varphi_{6}=\left(\hbar_{1}-2 \xi-\hbar_{2}\right), \xi=\max _{k \in \mathcal{N}}\left\{\xi_{k}\right\}, \\
& \underline{b}_{k h}^{l}=\min _{t \geq 0}\left\{b_{k h}^{l}(t)\right\}, \quad \hbar_{1}=\min _{k, h \in \mathcal{N}}\left\{2 N L \rho_{k}^{l} b_{k h}^{l}\right\}, \\
& \hbar_{2}=\max _{k, h \in \mathcal{N}}\left\{2 N L \sigma_{k}^{l} \tilde{b}_{k h}^{l}\right\}, \tilde{\rho}_{k}=\max _{l \in \mathcal{L}}\left\{\rho_{k}^{l}\right\}, \\
& \tilde{b}_{k h}^{l}=\max _{t \geq 0}\left\{b_{k h}^{l}(t)\right\}, \bar{d}=\max _{k \in \mathcal{N}}\left\{d_{k}\right\}, \underline{d}=\min _{k \in \mathcal{N}}\left\{d_{k}\right\}, \\
& \zeta=\max _{k \in N}\left\{2 \xi_{k}+\sum_{l=1}^{L} \sum_{h=1}^{N}\left(2 \rho_{k}^{l}+\sigma_{k}^{l}\right) b_{k h}^{l}(t)+L \sum_{h=1}^{N} Q_{k h}\right\}, \\
& \omega=\left(1+\frac{\varphi_{2}}{\beta}\right) e^{\ell} V\left(e\left(t_{s}\right), t_{s}\right), \lambda_{2}^{s}=1+\frac{\varphi_{2}}{\beta}+\frac{\varphi_{4} e^{\beta T_{s}}}{1-\varphi_{3} e^{\beta T_{s}}}, \\
& \ell=\max _{k \in \mathcal{N}}\left\{\left(2 \xi_{k}-2 a_{k}+2 \lambda_{1}+L \sum_{h=1}^{N} Q_{k h}\right) T_{s}\right. \\
& \left.+\left(2 \tilde{\rho}_{k}+\tilde{\sigma}_{k}\right) L N \varepsilon\right\}, \tilde{\sigma}_{k}=\max _{l \in \mathcal{L}}\left\{\sigma_{k}^{l}\right\} .
\end{aligned}
$$

Lemma 3.1: Assume that Assumptions 1-4 hold. If $0<$ $\varphi_{3}<1$ and $\beta \in\left[0,-\frac{\ln \varphi_{3}}{T_{s}}\right)$, the following inequality can be derived

$$
V(e(t), t) \leq k_{3} e^{-\beta\left(t-t_{s}\right)}, t \in\left[t_{s}, t_{s+1}^{\prime}\right),
$$

where $k_{1}=\frac{\omega}{\varphi_{3}}, k_{2}=\frac{\varphi_{5} e^{\beta T_{s}}}{1-\varphi_{3} e^{\beta T_{s}}}$ and $k_{3}=k_{1}+k_{2}$.

Proof: Define Lyapunov function $V(e(t), t)=$ $\sum_{k=1}^{N} d_{k} V_{k}\left(e_{k}(t), t\right)$, where $V_{k}\left(e_{k}(t), t\right)=\left|e_{k}(t)\right|^{2}$ and $d_{k}$ is the cofactor of the $k$ th diagonal element of the Laplacian matrix of digraph $(\mathcal{K}, \mathbb{B})$. When $t \in\left[t_{s}, t_{s+1}^{\prime}\right)$, consider measurement error, the error system (5) can be formulated as

$$
\begin{aligned}
\mathrm{d} e_{k}(t)= & {\left[f_{k}\left(y_{k}(t), t\right)-f_{k}\left(x_{k}(t), t\right)+\sum_{l=1}^{L} \sum_{h=1}^{N} b_{k h}^{l}(t) H_{k h l}\left(e_{k}(t), e_{h}(t)\right)\right.} \\
& \left.-a_{k}\left(e_{k}(t)-E_{k}(t)\right)\right] \mathrm{d} t .
\end{aligned}
$$

According to Assumption 1, one can obtain

$$
e_{k}^{\mathrm{T}}(t)\left[f_{k}\left(y_{k}(t), t\right)-f_{k}\left(x_{k}(t), t\right)\right] \leq \xi_{k}\left|e_{k}(t)\right|^{2} .
$$

On account of the Young's inequality $|a|^{\mathcal{A}}|b|^{\mathcal{B}} \leq$ $\frac{\mathcal{A}}{\mathcal{A}+\mathcal{B}}|a|^{\mathcal{A}+\mathcal{B}}+\frac{\mathcal{B}}{\mathcal{A}+\mathcal{B}}|b|^{\mathcal{A}+\mathcal{B}}$, where $\mathcal{A}, \mathcal{B}>0$, one can get

$$
\begin{aligned}
& 2 e_{k}^{\mathrm{T}}(t) \sum_{l=1}^{L} \sum_{h=1}^{N} b_{k h}^{l}(t)\left(H_{k h l}\left(y_{k}(t), y_{h}(t)\right)-H_{k h l}\left(x_{k}(t), x_{h}(t)\right)\right) \\
\leq & 2\left|e_{k}(t)\right| \sum_{l=1}^{L} \sum_{h=1}^{N} b_{k h}^{l}(t)\left(\rho_{k}^{l}\left|e_{k}(t)\right|+\sigma_{k}^{l}\left|e_{h}(t)\right|\right) \\
\leq & \sum_{l=1}^{L} \sum_{h=1}^{N}\left[\left(2 \rho_{k}^{l}+\sigma_{k}^{l}\right) b_{k h}^{l}(t)+Q_{k h}\right]\left|e_{k}(t)\right|^{2} \\
& +\sum_{l=1}^{L} \sum_{h=1}^{N} Q_{k h}\left(\left|e_{h}(t)\right|^{2}-\left|e_{k}(t)\right|^{2}\right)
\end{aligned}
$$

where $Q_{k h}=\max _{l \in L}\left\{\sigma_{k}^{l} \tilde{b}_{k h}^{l}\right\}$. Based on (8) and (9), we know that

$$
\begin{aligned}
& \frac{\mathrm{d} V(e(t), t)}{\mathrm{d} t} \\
\leq & \sum_{k=1}^{N} d_{k}\left[\left(2 \xi_{k}-2 a_{k}+\sum_{l=1}^{L} \sum_{h=1}^{N}\left(2 \rho_{k}^{l}+\sigma_{k}^{l}\right) b_{k h}^{l}(t)+L \sum_{h=1}^{N} Q_{k h}\right)\right. \\
& \left.\times\left|e_{k}(t)\right|^{2}+2 a_{k} e_{k}^{T} E_{k}(t)+L \sum_{h=1}^{N} Q_{k h}\left(\left|e_{h}(t)\right|^{2}-\left|e_{k}(t)\right|^{2}\right)\right] .
\end{aligned}
$$

Through computation, we have

$$
2 a_{k} e_{k}^{\mathrm{T}}(t) E_{k}(t) \leq 2 \lambda_{1}\left|e_{k}(t)\right|^{2}+\frac{a_{k}^{2}}{2 \lambda_{1}}\left|E_{k}(t)\right|^{2} .
$$

In view of Theorem 2.2 in [38], since $C_{\mathcal{Q}}$ denotes the directed cycle of $\mathcal{Q}$, one can get

$$
\begin{aligned}
& \sum_{k=1}^{N} \sum_{h=1}^{N} d_{k} Q_{k h}\left(\left|e_{h}(t)\right|^{2}-\left|e_{k}(t)\right|^{2}\right) \\
= & \sum_{\mathcal{Q} \in \mathbb{Q}} W(\mathcal{Q}) \sum_{(s, r) \in E\left(C_{\mathcal{Q}}\right)}\left(\left|e_{r}(t)\right|^{2}-\left|e_{s}(t)\right|^{2}\right) .
\end{aligned}
$$

Since $\sum_{(s, r) \in E\left(C_{\mathcal{Q}}\right)}\left(\left|e_{r}\right|^{2}-\left|e_{s}\right|^{2}\right)=0$, it can be obtained

$$
\sum_{k=1}^{N} \sum_{h=1}^{N} d_{k} Q_{k h}\left(\left|e_{h}(t)\right|^{2}-\left|e_{k}(t)\right|^{2}\right)=0
$$


Substituting (11), (12) into (10), we arrive at

$$
\begin{aligned}
& \frac{\mathrm{d} V(e(t), t)}{\mathrm{d} t} \\
\leq & \sum_{k=1}^{N} d_{k}\left[2 \xi_{k}-2 a_{k}+\sum_{l=1}^{L} \sum_{h=1}^{N}\left(2 \rho_{k}^{l}+\sigma_{k}^{l}\right) b_{k h}^{l}(t)\right. \\
& \left.+L \sum_{h=1}^{N} Q_{k h}+2 \lambda_{1}\right]\left|e_{k}(t)\right|^{2}+\sum_{k=1}^{N} d_{k} \frac{a_{k}^{2}}{2 \lambda_{1}}\left|E_{k}(t)\right|^{2}, \\
\leq & \sum_{k=1}^{N} d_{k}\left[2 \xi_{k}-2 a_{k}+\sum_{l=1}^{L} \sum_{h=1}^{N}\left(2 \rho_{k}^{l}+\sigma_{k}^{l}\right) b_{k h}^{l}(t)\right. \\
& \left.+L \sum_{h=1}^{N} Q_{k h}+2 \lambda_{1}\right]\left|e_{k}(t)\right|^{2}+\max _{k \in \mathcal{N}}\left\{\frac{d_{k} a_{k}^{2}}{2 \lambda_{1}}\right\} \sum_{k=1}^{N}\left|E_{k}(t)\right|^{2} .
\end{aligned}
$$

Besides, on account of (4), (13) can be interpreted as

$$
\frac{\mathrm{d} V(e(t), t)}{\mathrm{d} t} \leq \varphi_{1}(t) V(e(t), t)+\varphi_{2} V\left(e\left(t_{s}\right), t_{s}\right) e^{-\beta\left(t-t_{s}\right)},
$$

Then, it can be calculated that

$$
\begin{aligned}
V(e(t), t) \leq & V\left(e\left(t_{s}\right), t_{s}\right) e^{\int_{t_{s}}^{t} \varphi_{1}(\tau) \mathrm{d} \tau}+\int_{t_{s}}^{t} \varphi_{2} V\left(e\left(t_{s}\right), t_{s}\right) \\
& \times e^{-\beta\left(\tau-t_{s}\right)}\left(e^{\int_{\tau}^{t} \varphi_{1}(s) \mathrm{d} s}\right) \mathrm{d} \tau
\end{aligned}
$$

According to Assumption 4, when $t \in\left[t_{s}, t_{s}+T_{s}\right]$, one has $V(e(t), t) \leq \omega$, in which $\omega=\left(1+\frac{\varphi_{2}}{\beta}\right) e^{\ell} V\left(e\left(t_{s}\right), t_{s}\right)$. Similarly, when $t \in\left[t_{s}, t_{s+1}^{\prime}-T_{s}\right)$, it follows that

$$
\begin{aligned}
& V\left(e\left(t+T_{s}\right), t+T_{s}\right) \\
\leq & V(e(t), t) e^{\ell}+e^{\ell} \int_{t}^{t+T_{s}} \varphi_{2} V\left(e\left(t_{s}\right), t_{s}\right) e^{-\beta\left(\tau-t_{s}\right)} \mathrm{d} \tau \\
\leq & V(e(t), t) e^{\ell}+e^{\ell} \frac{\varphi_{2}}{\beta} V\left(e\left(t_{s}\right), t_{s}\right)\left(e^{-\beta\left(t-t_{s}\right)}-e^{-\beta\left(t+T_{s}-t_{s}\right)}\right) \\
\leq & V(e(t), t) e^{\ell}+e^{\ell} \frac{\varphi_{2}}{\beta} \cdot V\left(e\left(t_{s}\right), t_{s}\right) e^{-\beta\left(t-t_{s}\right)} \\
\leq & \varphi_{3} V(e(t), t)+\varphi_{4} V\left(e\left(t_{s}\right), t_{s}\right) e^{-\beta\left(t-t_{s}\right)} .
\end{aligned}
$$

Next, we will prove the following inequality holds

$$
V(e(t), t) \leq k_{1} e^{-\tilde{\alpha}\left(t-t_{s}\right)}+k_{2} e^{-\beta\left(t-t_{s}\right)},
$$

where $\tilde{\alpha}=\frac{-\ln \varphi_{3}}{T_{s}}, k_{1}=\frac{\omega}{\varphi_{3}}$ and $k_{2}=\frac{\varphi_{5} e^{\beta T_{s}}}{1-\varphi_{3} e^{\beta T_{s}}}$. In the following, for any $t>t_{s}$, we can find an integer $M$, such that $t_{s}+(M-1) T_{s} \leq t \leq t_{s}+M T_{s}$. Then, two cases are considered as follows:

case (i): when $t \geq t_{s}+T_{s}$, based on (14), one has

$$
\begin{aligned}
& V(e(t), t) \\
\leq & \varphi_{3} V\left(e\left(t-T_{s}\right), t-T_{s}\right)+\varphi_{5} e^{-\beta\left(t-T_{s}-t_{s}\right)} \\
\leq & \varphi_{3}^{2} V\left(e\left(t-2 T_{s}\right), t-2 T_{s}\right)+\varphi_{3} \varphi_{5} e^{-\beta\left(t-2 T_{s}-t_{s}\right)} \\
& +\varphi_{5} e^{-\beta\left(t-T_{s}-t_{s}\right)} \\
& \vdots \\
\leq & \varphi_{3}^{M-1} V\left(e\left(t-(M-1) T_{s}\right), t-(M-1) T_{s}\right) \\
& +\left[\varphi_{3}^{M-2} e^{\beta(M-1)}+\cdots+\varphi_{3} e^{2 \beta T_{s}}+e^{\beta T_{s}}\right] \varphi_{5} e^{-\beta\left(t-t_{s}\right)} .
\end{aligned}
$$

Since $t_{s}+M T_{s} \geq t$, it yields that $M \geq \frac{t-t_{s}}{T_{s}}$ and $t-(M-$ 1) $T_{s} \in\left[t_{s}, t_{s}+T_{s}\right)$. Thus,we can obtain that

$$
\varphi_{3}^{M-1} V\left(e\left(t-(M-1) T_{s}\right), t-(M-1) T_{s}\right) \leq \frac{\omega}{\varphi_{3}} e^{-\tilde{\alpha}\left(t-t_{s}\right)} .
$$

According to $\beta \in\left(0,-\frac{\ln \varphi_{3}}{T_{s}}\right)$, we have $\varphi_{3} e^{\beta} T_{s}<1$. Thereby,

$$
\begin{aligned}
& {\left[\varphi_{3}^{M-2} e^{(M-1) \beta T_{s}}+\cdots+\varphi_{3} e^{2 \beta T_{s}}+e^{\beta T}\right] \varphi_{5} e^{-\beta\left(t-t_{s}\right)}} \\
& \leq \varphi_{5} e^{-\beta\left(t-t_{s}\right)} \frac{e^{\beta T_{s}}\left(1-\left(\varphi_{3} e^{\beta T_{s}}\right)^{M-1}\right)}{1-\varphi_{3} e^{\beta T_{s}}} \\
& \quad \vdots \\
& \leq \frac{\varphi_{5} e^{\beta T_{s}}}{1-\varphi_{3} e^{\beta T_{s}}} e^{-\beta\left(t-t_{s}\right)}
\end{aligned}
$$

Substituting (16), (17) into (15) follows

$$
V(e(t), t) \leq \frac{\omega}{\varphi_{3}} e^{\tilde{\alpha}\left(t-t_{s}\right)}+\frac{\varphi_{5} e^{\beta T_{s}}}{1-\varphi_{3} e^{\beta T_{s}}} e^{-\beta\left(t-t_{s}\right)},
$$

case (ii): When $t_{s} \leq t<t_{s}+T_{s}$, it is easy to get

$$
V(e(t), t) \leq \omega \leq \omega e^{\tilde{\alpha}\left(t_{s}+T_{s}\right)} e^{-\tilde{\alpha} t}=\frac{\omega}{\varphi_{3}} e^{\tilde{\alpha}\left(t-t_{s}\right)}
$$

Hence, combining the above two cases, we get

$$
\begin{aligned}
V(e(t), t) & =\frac{\omega}{\varphi_{3}} e^{-\tilde{\alpha}\left(t-t_{s}\right)}+\frac{\varphi_{5} e^{\beta T}}{1-\varphi_{3} e^{\beta T}} e^{-\beta\left(t-t_{s}\right)} \\
& \leq k_{1} e^{-\tilde{\alpha}\left(t-t_{s}\right)}+k_{2} e^{-\beta\left(t-t_{s}\right)} \\
& \leq k_{3} e^{-\beta\left(t-t_{s}\right)}
\end{aligned}
$$

The proof is completed.

Lemma 3.2: Suppose that all the conditions in Lemma 1 hold. There exists a constant $T^{*}>0$ such that $t_{s+1}^{\prime}-t_{s} \geq T^{*}$ for $s=0,1,2, \ldots$ That is to say, Zeno behavior can be avoid under the event-triggered conditions (4).

Proof: When $t \in\left[t_{s}, t_{s+1}^{\prime}\right)$, it follows from measurement error that $E(t)=e(t)-e\left(t_{s}\right)$. Then, we can rewrite system (5) as follows

$$
\begin{aligned}
\mathrm{d} E_{k}(t)= & {\left[f_{k}\left(e_{k}(t), t\right)+\sum_{l=1}^{L} \sum_{h=1}^{N} b_{k h}^{l}(t) H_{k h l}\left(e_{k}(t), e_{h}(t)\right)\right.} \\
& \left.-a_{k}\left(y_{k}\left(t_{s}\right)-x_{k}\left(t_{s}\right)\right)\right] \mathrm{d} t .
\end{aligned}
$$

Therefore, one can get

$$
\begin{aligned}
\mathrm{d}|E(t)|^{2}= & \sum_{k=1}^{N} 2 E_{k}^{\mathrm{T}}(t)\left[f_{k}\left(e_{k}(t), t\right)+\sum_{l=1}^{L} \sum_{h=1}^{N} b_{k h}^{l}(t) H_{k h l}\left(e_{k}(t), e_{h}(t)\right)\right. \\
& \left.-a_{k}\left(y_{k}\left(t_{s}\right)-x_{k}\left(t_{s}\right)\right)\right] \mathrm{d} t
\end{aligned}
$$

According to Assumption 1, it yields that

$$
\begin{aligned}
& 2 E_{k}^{\mathrm{T}}(t)\left(f_{k}\left(y_{k}(t), t\right)-f_{k}\left(x_{k}(t), t\right)\right) \\
\leq & \left|E_{k}(t)\right|^{2}+\left|f_{k}\left(e_{k}(t), t\right)\right|^{2} \\
\leq & \left|E_{k}(t)\right|^{2}+\xi_{k}^{2}\left|\left(e_{k}(t)\right)\right|^{2} .
\end{aligned}
$$


In view of Assumption 2, we have the following inequality

$$
\begin{aligned}
& 2 E_{k}^{\mathrm{T}}(t) \sum_{l=1}^{L} \sum_{h=1}^{N} b_{k h}^{l}(t) H_{k h l}\left(e_{k}(t), e_{h}(t)\right) \\
\leq & 2\left|E_{k}(t)\right| \sum_{l=1}^{L} \sum_{h=1}^{N} b_{k h}^{l}(t)\left(\rho_{k}^{l}\left|e_{k}(t)\right|+\sigma_{k}^{l}\left|e_{h}(t)\right|\right) \\
\leq & \sum_{l=1}^{L} \sum_{h=1}^{N} \tilde{b}_{k h}^{l}\left(\rho_{k}^{l}+\sigma_{k}^{l}\right)\left|E_{k}(t)\right|^{2}+\sum_{l=1}^{L} \sum_{h=1}^{N} \tilde{b}_{k h}^{l} \rho_{k}^{l}\left|e_{k}(t)\right|^{2} \\
& +\sum_{l=1}^{L} \sum_{h=1}^{N} \tilde{b}_{k h}^{l} \sigma_{k}^{l}\left|e_{h}(t)\right|^{2}
\end{aligned}
$$

Substituting (19), (20) into (18), we have

$$
\begin{aligned}
& \frac{\mathrm{d}|E(t)|^{2}}{\mathrm{~d} t} \\
\leq & \sum_{k=1}^{N}\left[\left|E_{k}(t)\right|^{2}+\xi_{k}^{2}\left|\left(e_{k}(t)\right)\right|^{2}+\sum_{l=1}^{L} \sum_{h=1}^{N} \tilde{b}_{k h}^{l}\left(\rho_{k}^{l}+\sigma_{k}^{l}\right)\right. \\
& \times\left|E_{k}(t)\right|^{2}+\sum_{l=1}^{L} \sum_{h=1}^{N} \tilde{b}_{k h}^{l} \rho_{k}^{l}\left|e_{k}(t)\right|^{2}+\sum_{l=1}^{L} \sum_{h=1}^{N} \tilde{b}_{k h}^{l} \sigma_{k}^{l}\left|e_{h}(t)\right|^{2} \\
& \left.-2 a_{k} E_{k}^{\mathrm{T}}(t) e_{k}\left(t_{s}\right)\right] \\
\leq & \max _{k, h \in \mathcal{N}, l \in \mathcal{L}}\left\{1+\xi_{k}^{2}+2 L N \tilde{b}_{k h}^{l}\left(\rho_{k}^{l}+\sigma_{k}^{l}\right)+a_{k}\right\}|E(t)|^{2} \\
& +\underset{k, h \in \mathcal{N}, l \in \mathcal{L}}{\max _{k}}\left\{\xi_{k}^{2}+L N \tilde{b}_{k h}^{l}\left(\rho_{k}^{l}+\sigma_{k}^{l}\right)+a_{k}\right\}\left|e\left(t_{s}\right)\right|^{2} \\
\triangleq & \tilde{\rho}_{1}|E(t)|^{2}+\tilde{\rho}_{2}\left|e\left(t_{s}\right)\right|^{2} .
\end{aligned}
$$

Noting that $E\left(t_{s}\right)=0$, by utilizing Gronwall inequality, we have

$$
|E(t)|^{2} \leq \frac{\tilde{\rho}_{2}\left|e\left(t_{s}\right)\right|^{2}}{\tilde{\rho}_{1}}\left(e^{\tilde{\rho}_{1}\left(t-t_{s}\right)}-1\right) .
$$

Since $t_{s+1}^{\prime}=\inf \left\{t>t_{s}:|E(t)|^{2}-\delta_{2}\left|e\left(t_{s}\right)\right|^{2} e^{-\beta\left(t-t_{s}\right)} \geq 0\right\}$ we see that the next event will not be triggered before $|E(t)|^{2}=\delta_{2}\left|e\left(t_{s}\right)\right|^{2} e^{-\beta\left(t-t_{s}\right)}$. Then, combined with (21), one has

$$
\delta_{2}\left|e\left(t_{s}\right)\right|^{2} e^{-\beta\left(t-t_{s}\right)} \leq \frac{\tilde{\rho}_{2}\left|e\left(t_{s}\right)\right|^{2}}{\tilde{\rho}_{1}}\left(e^{\tilde{\rho}_{1}\left(t-t_{s}\right)}-1\right) .
$$

Thus, for any $t \in\left[t_{s}, t_{s+1}^{\prime}\right)$

$$
\delta_{2}\left|e\left(t_{s}\right)\right|^{2} e^{-\beta\left(t-t_{s}\right)}+\frac{\tilde{\rho}_{2}\left|e\left(t_{s}\right)\right|^{2}}{\tilde{\rho}_{1}} \leq \frac{\tilde{\rho}_{2}\left|e\left(t_{s}\right)\right|^{2}}{\tilde{\rho}_{1}} e^{\tilde{\rho}_{1}\left(t-t_{s}\right)} .
$$

Thus, the following is satisfied

$$
t_{s+1}^{\prime}-t_{s} \geq \frac{\ln \left(1+\frac{\delta_{2} \tilde{\rho}_{1}}{\tilde{\rho}_{2}}\right)}{\tilde{\rho}_{1}+\beta} \triangleq T^{*} .
$$

Therefore, the Zeno behavior is avoided. This proof is finished.

Theorem 3.1: If Assumptions 1-4 hold, and

$$
\begin{aligned}
& e_{k}^{\mathrm{T}}(t) H_{k h l}\left(e_{k}(t), e_{h}(t)\right) \geq \tilde{\rho}_{k}^{l}\left|e_{k}(t)\right|^{2}-\tilde{\sigma}_{k}^{l}\left|e_{h}(t)\right|^{2}, \\
& \zeta\left(\frac{\ln \left(2+2 \delta_{1}\right)}{\varphi_{6}-\alpha}\right) \leq \frac{\beta}{3}\left(\frac{\ln \left(1+\frac{\delta_{2} \tilde{\rho}_{1}}{\tilde{\rho}_{2}}\right)}{\tilde{\rho}_{1}+\beta}\right)-\ln \lambda_{2}^{S},
\end{aligned}
$$

where $0<\varphi_{3}<1, \beta \in\left[0,-\frac{\ln \varphi_{3}}{T_{s}}\right)$ and $\varphi_{6}>\alpha$, then drive-response systems (1) and (2) achieve exponential synchronization. have

Proof: Let $\nu(t)=-|e(t)|^{2}$, when $t \in\left[t_{s+1}^{\prime}, t_{s+1}\right)$, we

$$
\begin{aligned}
& \frac{\mathrm{d} \nu(t)}{\mathrm{d} t} \\
= & \sum_{k=1}^{N}-2 e_{k}^{\mathrm{T}}(t)\left[f_{k}\left(e_{k}(t), t\right)+\sum_{l=1}^{L} \sum_{h=1}^{N} b_{k h}^{(l)}(t) H_{k h l}\left(e_{k}(t), e_{h}(t)\right)\right] \\
\leq & \sum_{k=1}^{N}\left[2 \xi_{k}\left|e_{k}(t)\right|^{2}-\sum_{l=1}^{L} \sum_{h=1}^{N} 2 \rho_{k}^{l} \underline{b}_{k h}^{l}\left|e_{k}(t)\right|^{2}+\sum_{l=1}^{L} \sum_{h=1}^{N} 2 \tilde{b}_{k h}^{l} \sigma_{k}^{l}\left|e_{h}(t)\right|^{2}\right] \\
\leq & \left(\hbar_{1}-2 \xi-\hbar_{2}\right) \nu(t)=\varphi_{6} \nu(t) .
\end{aligned}
$$

Thus, we can obtain that

$$
|e(t)|^{2} \geq\left|e\left(t_{s+1}^{\prime}\right)\right|^{2} e^{\varphi_{6}\left(t-t_{s+1}^{\prime}\right)}
$$

Based on event triggering function (4), the next event will not be triggering until $|N(t)|^{2}=\delta_{1}\left|e\left(t_{s+1}^{\prime}\right)\right|^{2} \exp \left\{\alpha\left(t-t_{s+1}^{\prime}\right)\right\}$. Hence, based on elementary inequality and $N(t)=e(t)-$ $e\left(t_{s+1}^{\prime}\right)$, it yields that

$$
\frac{1}{2}|e(t)|^{2}-\left|e\left(t_{s+1}^{\prime}\right)\right|^{2}-\delta_{1}\left|e\left(t_{s+1}^{\prime}\right)\right|^{2} e^{\alpha\left(t-t_{s+1}^{\prime}\right)} \leq 0 .
$$

Then, combined with (23), we can get

$$
\frac{1}{2}\left|e\left(t_{s+1}^{\prime}\right)\right|^{2} e^{\varphi_{6}\left(t_{s+1}-t_{s+1}^{\prime}\right)}-\left|e\left(t_{s+1}^{\prime}\right)\right|^{2}-\delta_{1}\left|e\left(t_{s+1}^{\prime}\right)\right|^{2} e^{\alpha\left(t_{s+1}-t_{s+1}^{\prime}\right)} \leq 0
$$

Consequently, we have

$$
\frac{\ln \left(2+2 \delta_{1}\right)}{\varphi_{6}-\alpha} \geq t_{s+1}-t_{s+1}^{\prime} .
$$

When $t \in\left[t_{s+1}^{\prime}, t_{s+1}\right)$, according to (9), it is derived that

$$
\begin{aligned}
\frac{\mathrm{d} V(e(t), t)}{\mathrm{d} t} & \leq \sum_{k=1}^{N} \mathrm{~d}_{k}(t)\left(2 \xi_{k}+\sum_{l=1}^{L} \sum_{h=1}^{N}\left(2 \rho_{k}^{l}+\sigma_{k}^{l}\right) b_{k h}^{l}(t)+L \sum_{h=1}^{N} Q_{k h}\right) \\
& \times\left|e_{k}(t)\right|^{2}+L \sum_{k=1}^{N} \sum_{h=1}^{N} d_{k} Q_{k h}\left(\left|e_{h}(t)\right|^{2}-\left|e_{k}(t)\right|^{2}\right) \\
& \leq \zeta V(e(t), t)
\end{aligned}
$$

Consequently, we have

$$
V(e(t), t) \leq V\left(e\left(t_{s+1}^{\prime}\right), t_{s+1}^{\prime}\right) e^{\zeta\left(t-t_{s+1}^{\prime}\right)}
$$

When $t \in\left[t_{s}, t_{s+1}^{\prime}\right)$, in view of Lemma 1 , it is clear that

$$
\begin{aligned}
& V(e(t), t) \\
\leq & \left(1+\frac{\varphi_{2}}{\beta}\right) V\left(e\left(t_{s}\right), t_{s}\right) e^{-\tilde{\alpha}\left(t-t_{s}\right)}+\frac{\varphi_{4} e^{\beta T_{s}}}{1-\varphi_{3} e^{\beta T_{s}}} V\left(e\left(t_{s}\right), t_{s}\right) e^{-\beta\left(t-t_{s}\right)} \\
\leq & \left(1+\frac{\varphi_{2}}{\beta}+\frac{\varphi_{4} e^{\beta T_{s}}}{1-\varphi_{3} e^{\beta T_{s}}}\right) V\left(e\left(t_{s}\right), t_{s}\right) e^{-\beta\left(t-t_{s}\right)} \\
= & V\left(e\left(t_{s}\right), t_{s}\right) e^{\left(-\beta\left(t-t_{s}\right)+\ln \lambda_{2}^{s}\right)}
\end{aligned}
$$

Consequently, when $t \in\left[t_{0}, t_{1}^{\prime}\right)$, we have

$$
V(e(t), t) \leq V\left(e\left(t_{0}\right), t_{0}\right) e^{\left(-\beta\left(t-t_{0}\right)+\ln \lambda_{2}^{0}\right)} .
$$


When $t \in\left[t_{1}^{\prime}, t_{1}\right)$,

$$
\begin{aligned}
V(e(t), t) & \leq V\left(e\left(t_{1}^{\prime}\right), t_{1}^{\prime}\right) e^{\zeta\left(t-t_{1}^{\prime}\right)} \\
& \leq V\left(e\left(t_{0}\right), t_{0}\right) e^{\left(\zeta\left(t-t_{1}^{\prime}\right)+\ln \lambda_{2}^{0}-\beta\left(t-t_{0}\right)\right)} .
\end{aligned}
$$

When $t \in\left[t_{1}, t_{2}^{\prime}\right)$,

$$
\begin{aligned}
V(e(t), t) & \leq V\left(e\left(t_{1}\right), t_{1}\right) e^{\left(-\beta\left(t-t_{1}\right)+\ln \lambda_{2}^{1}\right)} \\
& \leq V\left(e\left(t_{0}\right), t_{0}\right) e^{\left(-\beta\left(t-t_{1}\right)+\ln \lambda_{2}^{1}+\zeta\left(t-t_{1}^{\prime}\right)+\ln \lambda_{2}^{0}-\beta\left(t-t_{0}\right)\right)} .
\end{aligned}
$$

When $t \in\left[t_{2}^{\prime}, t_{2}\right)$,

$$
\begin{aligned}
& V(e(t), t) \\
\leq & V\left(e\left(t_{2}^{\prime}\right), t_{2}^{\prime}\right) e^{\zeta\left(t-t_{2}^{\prime}\right)} \\
\leq & V\left(e\left(t_{0}\right), t_{0}\right) e^{\left(\zeta\left(t-t_{2}^{\prime}\right)-\beta\left(t-t_{1}\right)+\ln \lambda_{2}^{1}+\zeta\left(t-t_{1}^{\prime}\right)+\ln \lambda_{2}^{0}-\beta\left(t-t_{0}\right)\right)} .
\end{aligned}
$$

Through mathematical induction, when $t \in\left[t_{s+1}^{\prime}, t_{s+1}\right)$, one has

$$
\begin{aligned}
& V(e(t), t) \\
\leq & V\left(e\left(t_{s+1}^{\prime}\right), t_{s+1}^{\prime}\right) e^{\zeta\left(t-t_{s+1}^{\prime}\right)} \\
\leq & V\left(e\left(t_{s}\right), t_{s}\right) e^{\left(\zeta\left(t-t_{s+1}^{\prime}\right)+\ln \lambda_{2}^{s}-\beta\left(t_{s+1}^{\prime}-t_{s}\right)\right)} \\
\leq & V\left(e\left(t_{0}\right), t_{0}\right) e^{\left(\zeta\left(\left(t-t_{s+1}^{\prime}\right)+\sum_{j=1}^{s}\left(t_{j}-t_{j}^{\prime}\right)\right)+\sum_{j=0}^{s} \ln \lambda_{2}^{j}-\sum_{j=0}^{s} \beta\left(t_{j+1}^{\prime}-t_{j}\right)\right)} .
\end{aligned}
$$

Then, it follows from (22) and (24) that the following inequality holds

$$
\zeta\left(t_{s+1}-t_{s+1}^{\prime}\right) \leq \frac{\beta}{3}\left(t_{s+1}^{\prime}-t_{s}\right)-\ln \lambda_{2}^{S} .
$$

Thus, we can obtain that

$$
\begin{aligned}
& \zeta\left(t_{s+1}-t_{s+1}^{\prime}\right)+\ln \lambda_{2}^{S}-\beta\left(t_{s+1}^{\prime}-t_{s}\right) \\
\leq & -\frac{2 \beta}{3}\left(t_{s+1}^{\prime}-t_{s}\right) \\
\leq & -\zeta\left(t_{s+1}-t_{s+1}^{\prime}\right)-\ln \lambda_{2}^{S}-\frac{\beta}{3}\left(t_{s+1}^{\prime}-t_{s}\right) \\
\leq & -\Lambda\left(t_{s+1}^{\prime}-t_{s}\right),
\end{aligned}
$$

where $\Lambda=\min \left\{\zeta, \frac{\beta}{3}\right\}$. Combined with (26), it can be derived that

$$
\begin{aligned}
& \xi\left(\left(t-t_{s+1}^{\prime}\right)+\sum_{j=1}^{s}\left(t_{j}-t_{j}^{\prime}\right)\right)+\sum_{j=0}^{s} \ln \lambda_{2}^{j}-\sum_{j=0}^{s} \beta\left(t_{j+1}^{\prime}-t_{j}\right. \\
\leq & -\Lambda\left(t-t_{0}\right),
\end{aligned}
$$

As a consequence, when $t \in\left[t_{s+1}^{\prime}, t_{s+1}\right)$, one has

$$
V(e(t), t) \leq V\left(e\left(t_{0}\right), t_{0}\right) e^{-\Lambda\left(t-t_{0}\right)} .
$$

When $t \in\left[t_{s}, t_{s+1}^{\prime}\right)$, we can obtain

$$
\begin{aligned}
& V(e(t), t) \\
\leq & V\left(e\left(t_{s}\right), t_{s}\right) e^{\left(-\beta\left(t-t_{s}\right)+\ln \lambda_{2}^{S}\right)} \\
\leq & V\left(e\left(t_{0}\right), t_{0}\right) e^{\left(-\beta\left(t-t_{s}\right)+\sum_{j=1}^{s} \zeta\left(t_{j}-t_{j}^{\prime}\right)+\sum_{j=0}^{s} \ln \lambda_{2}^{s}-\sum_{j=0}^{s-1} \beta\left(t_{j+1}^{\prime}-t_{j}\right)\right) .}
\end{aligned}
$$

Similarly, one gets

$$
\begin{aligned}
& \quad-\beta\left(t-t_{s}\right)+\sum_{j=1}^{s} \zeta\left(t_{j}-t_{j}^{\prime}\right)+\sum_{j=0}^{s} \ln \lambda_{2}^{s}-\sum_{j=0}^{s-1} \beta\left(t_{j+1}^{\prime}-t_{j}\right) \\
\leq & -\Lambda\left(t-t_{0}\right),
\end{aligned}
$$

Thus, when $t \in\left[t_{s}, t_{s+1}^{\prime}\right)$, it yields

$$
V(e(t), t) \leq V\left(e\left(t_{0}\right), t_{0}\right) e^{-\Lambda\left(t-t_{0}\right)},
$$

Moreover, consider that

$$
V(e(t), t)=\sum_{k=1}^{N} d_{i}\left|e_{i}(t)\right|^{2} \geq \underline{d}|e(t)|^{2} .
$$

we finally obtain that

$$
|e(t)|^{2} \leq \frac{V\left(e\left(t_{0}\right), t_{0}\right)}{\underline{d}} e^{-\Lambda\left(t-t_{0}\right)} .
$$

According to Definition 1, we can derive that drive-response systems (1) and (2) achieve exponential synchronization. This completes the proof.

Remark 4: Although AIC is considered to be an effective control strategy and is set aperiodically in previous studies [24]- [26], however, assumptions that $(1) \inf _{i \in N}\left(s_{i}-t_{i}\right)=$ $\theta,(2) \sup _{i \in N}\left(t_{i+1}-t_{i}\right)=\omega$ or the maximum proportion of rest intervals are unavoidable, implying that the distributions of control and rest intervals are essentially uniformed. Fortunately, by designing a new type of control AETAIC, the conservativeness of traditional AIC is reduced and more complete aperiodicity are realized. Thus, the control we design is more flexible and of great practical significance.

\section{ApPlications And nUmerical eXAmples}

\subsection{Application to Chua's circuits}

Being one of the simplest autonomous circuits which can show the dynamical behaviors like bifurcation and chaos, Chua's circuits have attracted wide attention from investigators [12], [41]- [44]. In [42], authors propose a novel image compression-encryption scheme with good security performance based on Chua's circuit system. In [12], Chua's circuits are modeled on signed networks and the exponential bipartite $j$ synchronization problem is discussed. In this paper, a modified
Chua's circuit is given by

$$
\left\{\begin{array}{l}
\dot{V}_{1}(t)=-\frac{1}{l C_{1}} V_{1}(t)+\frac{1}{l C_{1}} V_{2}(t)-\frac{1}{C_{1}} \hat{f}\left(V_{1}(t)\right), \\
\dot{V}_{2}(t)=\frac{1}{l C_{2}} V_{1}(t)-\frac{1}{l C_{2}} V_{2}(t)+\frac{1}{C_{2}} i_{3}(t) \\
\dot{i}_{3}(t)=-\frac{1}{\tilde{M}}\left(V_{2}(t)+l_{0} i_{3}(t)\right)
\end{array}\right.
$$

where $V_{1}, V_{2}$ denote the voltage across $C_{1}$ and $C_{2}$, respectively and $i_{3}$ is the current through inductance $\tilde{M}$. What's more, $l$ and $l_{0}$ are linear resistors. And $\hat{f}\left(V_{1}\right)=G_{e} V_{1}+0.5\left(G_{d}-\right.$ $\left.G_{e}\right)\left(\left|V_{1}+1\right|-\left|V_{1}-1\right|\right)$ where $G_{d}$ and $G_{e}$ are the slopes of the inner and outer regions, respectively, is the current through 
the nonlinear resistor $N_{R}$. Considering coupling factor, system (27) can be depicted as

$\mathrm{d} X_{k}(t)=\left(\tilde{f}_{k}\left(X_{k}(t), t\right)+\sum_{l=1}^{L} \sum_{h=1}^{N} b_{k h}^{l} H_{k h}\left(X_{k}(t), X_{h}(t)\right)\right) \mathrm{d} t$

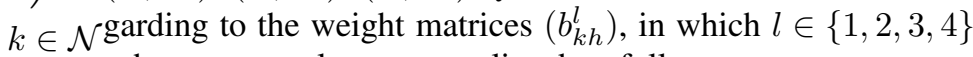

in which $X_{k}(t)=\left(x_{k 1}(t), x_{k 2}(t), x_{k 3}(t)\right)^{\mathrm{T}}, X_{i 1}(t)=$ $V_{1}(t), X_{i 2}(t)=V_{2}(t), X_{i 3}(t)=i_{3}(t), \Omega=\frac{1}{l C_{1}}, \Gamma=\frac{1}{l C_{2}}$, $\phi=\frac{1}{C_{2}}, \psi=\frac{1}{\tilde{M}}, \varphi=\frac{L_{0}}{\tilde{M}}, \varpi=\frac{1}{C_{1}}$ and

$$
\begin{aligned}
\tilde{f}\left(X_{k}(t), t\right)= & \left(\begin{array}{ccc}
-\Omega & \Omega & 0 \\
\Gamma & -\Gamma & \phi \\
0 & -\psi & -\varphi
\end{array}\right)\left(\begin{array}{l}
x_{k 1}(t) \\
x_{k 2}(t) \\
x_{k 3}(t)
\end{array}\right) \\
& +\left(\begin{array}{c}
-\varpi \hat{f}\left(x_{k 1}(t)\right) \\
0 \\
0
\end{array}\right) .
\end{aligned}
$$

And $H_{k h l}\left(X_{k}, X_{h}\right)$ is a coupling function satisfying the assumption $H_{k h l}\left(X_{k}, X_{h}\right) \leq \tilde{\rho}_{k}^{l}\left|X_{k}\right|+\tilde{\sigma}_{k}^{l}\left|X_{h}\right|$. Now, consider system (28) as the drive system, then the corresponding response system is given by

$$
\begin{aligned}
\mathrm{d} Y_{k}(t)= & \left(\hat{f}_{k}\left(Y_{k}(t), t\right)+\sum_{l=1}^{L} \sum_{h=1}^{N} b_{k h}^{l} H_{k h}\left(Y_{k}(t), Y_{h}(t)\right) \mathrm{d} t\right. \\
& \left.+U_{k}(t)\right) \mathrm{d} t, k \in \mathcal{N}
\end{aligned}
$$

where $Y_{k}(t)=\left(y_{k_{1}}(t), y_{k_{2}}(t), y_{k_{3}}(t)\right)^{\mathrm{T}}$ and $U_{k}$ is defined in (3). Moreover, set $E_{k}(t)=Y_{k}(t)-X_{k}(t)$, then the following error system is obtained

$$
\begin{aligned}
\mathrm{d} E_{k}(t)= & \left(\hat{f}_{k}\left(y_{k}(t), t\right)-\hat{f}_{k}\left(x_{k}(t), t\right)\right)+\sum_{l=1}^{L} \sum_{h=1}^{N} b_{k h}^{l} \\
& \left.H_{k h l}\left(E_{k}(t), E_{h}(t)\right)+U_{k}(t)\right) \mathrm{d} t, k \in \mathcal{N}
\end{aligned}
$$

in which $\quad E_{k}(t)=\left(e_{k_{1}}(t), e_{k_{2}}(t), e_{k_{3}}(t)\right)^{\mathrm{T}}$, $H_{k h l}\left(E_{k}(t), E_{h}(t)\right) \quad=\quad H_{k h l}\left(Y_{k}(t), Y_{h}(t)\right) \quad-$ $H_{k h l}\left(X_{k}(t), X_{h}(t)\right)$. According to Assumption 2, the function $\hat{f}$ satisfies $\hat{f}\left(e_{k 1}(t)\right)=\sigma\left(y_{k 1}(t)-x_{k 1}(t)\right)=\sigma e_{k 1}(t)$ with $G_{d} \leq \sigma \leq G_{e}$. Then one can derived that

$$
\begin{aligned}
& E_{k}^{\mathrm{T}}(t) \hat{f}_{k}\left(E_{k}(t), t\right) \\
= & e_{k}^{\mathrm{T}}(t)\left(\begin{array}{ccc}
-\Omega & \Omega & 0 \\
\Gamma & -\Gamma & \phi \\
0 & -\psi & -\varphi
\end{array}\right) e_{k}(t)+e_{k}^{T}(t)\left(\begin{array}{c}
-\hat{f}\left(e_{i 1}(t)\right) \\
0 \\
0
\end{array}\right) \\
= & -\Omega e_{k_{1}}^{2}(t)+\Gamma e_{k_{1}}(t) e_{k_{2}}(t)+\Omega e_{k_{1}}(t) e_{k_{2}}(t)-\Gamma e_{k_{2}}^{2}(t) \\
\leq & \left(-\gamma e_{k_{3}}(t) e_{k_{2}}(t)+\phi e_{k_{3}}(t) e_{k_{2}}-\varphi e_{k_{3}}^{2}(t)-\sigma e_{k_{1}}^{2}(t)\right. \\
& \left(-\frac{\Gamma}{2}+\frac{\Omega}{2}-\sigma\right) e_{k_{1}}^{2}(t)+\left(\frac{\Gamma}{2}+\frac{\Omega}{2}-\Gamma+\frac{\psi}{2}+\frac{\phi}{2}\right) \\
& \times e_{k_{2}}^{2}(t)+\left(\frac{\psi}{2}+\frac{\phi}{2}-\varphi\right) e_{k_{3}}^{2}(t) \\
= & \tilde{Q}\left|e_{k}(t)\right|^{2}
\end{aligned}
$$

where $\tilde{Q}=\max \left\{\frac{\Gamma-\Omega}{2}-\sigma, \frac{\Omega+\psi+\phi-\Gamma}{2}, \frac{\psi+\phi}{2}-\varphi\right\}$.
To verify the conditions in Theorem 1, we then consider a numerical example that the topological structure of MWCNs with 5 nodes is depicted in Figure 2 and each node on digraph $\mathcal{K}$ denotes a single Chua's circuit, where sub-digraphs $\left(\mathcal{K}, \mathbb{B}_{1}\right)$, , the nonzero elements are listed as follows.

- $b_{1,4}^{2}=b_{2,1}^{3}=b_{3,5}^{2}=0.001\left(0.2 e^{-t}+0.2\right) ; b_{2,4}^{4}=b_{3,2}^{1}=$ $0.001\left(0.2 e^{-t}+0.3\right)$

- $b_{4,3}^{1}=b_{3,2}^{3}=0.0001\left(0.1 e^{-t}+0.08\right) ; b_{5,2}^{2}=b_{5,2}^{3}=$ $0.001\left(0.5 e^{-t}+0.2\right)$

- $b_{3,1}^{1}=0.0001\left(0.09 e^{-t}+0.01\right) ; b_{4,3}^{2}=0.001\left(0.4 e^{-t}+\right.$ $0.2) ; b_{4,5}^{4}=0.001\left(0.2 e^{-t}+0.1\right)$

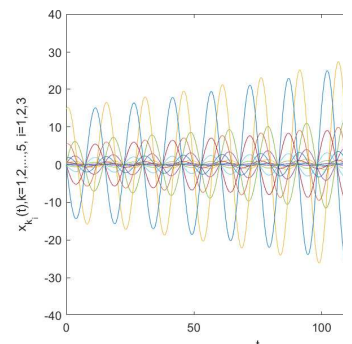

Fig. 3: Trajectories of drive system (28)

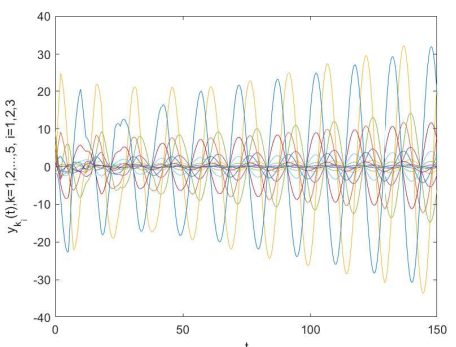

Fig. 4: Trajectories of response system (29)
Then set $l=5, l_{0}=0.25, C_{1}=1, C_{2}=2$, $\tilde{M}=2.5$. Besides, we choose control gain $a_{k_{1}}=0.81$, $a_{k_{2}}=0.82$ and $a_{k_{3}}=0.8$ and meanwhile, select the parameters of the event-triggered functions as $\alpha=0.1$, $\beta=0.24, \delta_{1}=0.8, \delta_{2}=5.2$ and the coupling functions as $H_{k h 1}\left(X_{k}(t), X_{h}(t)\right)=H_{k h 2}\left(X_{k}(t), X_{h}(t)\right)=$ $H_{k h 3}\left(X_{k}(t), X_{h}(t)\right)=H_{k h 4}\left(X_{k}(t), X_{h}(t)\right)=X_{k}-X_{h}$. Though calculation we obtain $\tilde{Q}=0.5>0$, then Assumption 1 and Theorem 1 can be both satisfied.

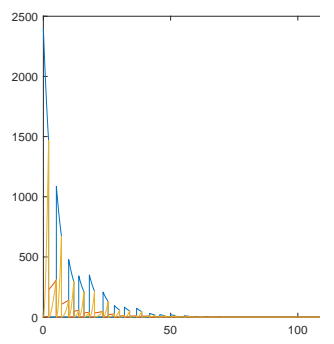

Fig. 5: Trajectories of triggering functions and measurement error

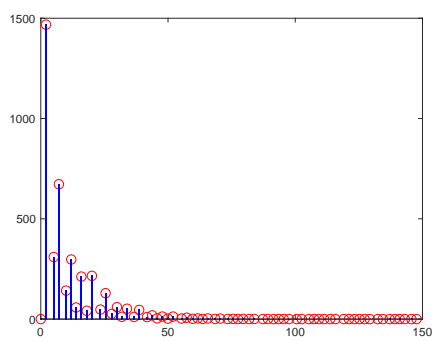

Fig. 6: The description of event-triggered time instants
For better illustration, trajectories of drive system (28) and response system (29) are exhibited in Figure 3 and Figure 4 respectively. The trajectories of measurement error and its corresponding triggering functions, as well as time instants are described in Figure 5 and Figure 6. Trajectories of the error system without AETAIC is presented in Figure 7 and it's clear 
to see that $E_{k}(t)$ do not tend to 0. Comparatively, from Figure 8 which depicts error system (30) with AETAIC, $E_{k}(t)$ tend to 0 under the designed control, whose trajectories are shown in Figure 9. Therefore, it can be inferred that the drive system and response system achieve exponential synchronization in mean square.

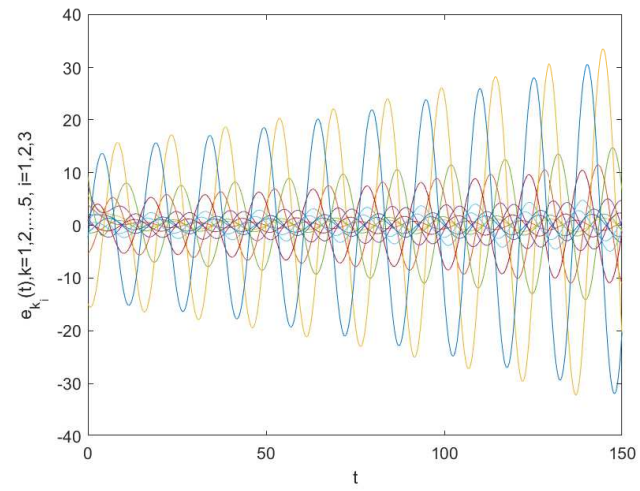

Fig. 7: Trajectories of error system (30) without control

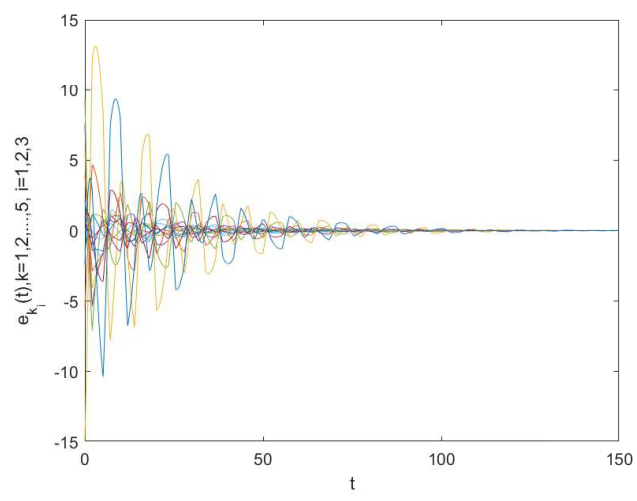

Fig. 8: Trajectories of error system (30) with control

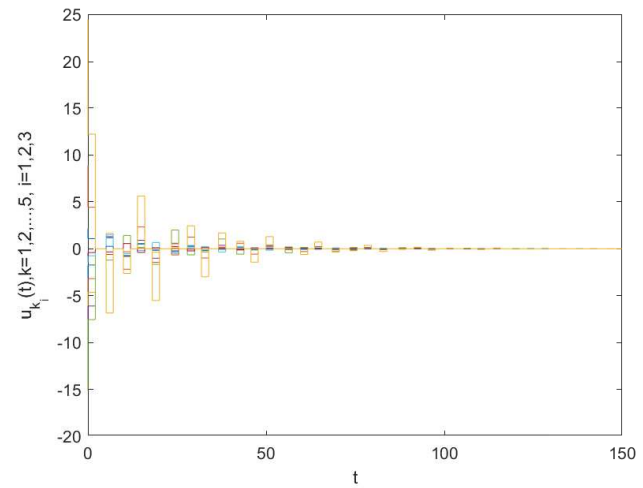

Fig. 9: Trajectories of control

Remark 5: Chua's circuit is a type of important nonlinear electronic circuits, which in real world can be connected not only in series but also in parallel. Thus, it is necessary to study Chua's circuits in networks with multi-links and extensive research has been carried out [12], [41]- [44]. For instance, in [12] Chua's circuits are modeled on signed networks with multi-links via aperiodically intermittent control. Also, the synchronization of stochastic hybrid Chua's circuits networks is addressed in [43], where mixed delays and multiple weights are considered. In this paper, we investigate MWCNs on Chua's circuit via a novel type of control AETAIC, which is different from previous work. Note that event-triggered AETAIC shows more complete aperiodicity and flexibility. Despite the advantages, there are few research employing AETAIC on MWCNs, let alone Chua's circuits. Thus, the results in this paper enrich the study of Chua's circuits.

\subsection{Application to coupled oscillators}

Nowadays, intensive research on coupled oscillators has been carried out owing to their wide applications in real world [45], [46]. In the following, we discuss coupled oscillators on multi-weighted complex networks. Initially, the second-order oscillators are presented as

$$
\ddot{x}_{k}(t)+\tilde{\rho} \dot{x}_{k}(t)+\xi_{k} x_{k}(t)=0, k \in \mathcal{N},
$$

in which $\tilde{\rho}$ is positive constant, $\xi_{k}>0, b_{k h}^{l}(t)$ are the weights of the edges between node $k$ and node $h$. Set $x_{k}^{*}(t)=\dot{x}_{k}(t)+$ $\tilde{\mu} x_{k}(t)$ where $\tilde{\mu}>0$, and system (31) can be rewritten in the following form

$$
\left\{\begin{aligned}
\mathrm{d} x_{k}(t)= & {\left[x_{k}^{*}(t)-\tilde{\mu} x_{k}(t)+\sum_{l=1}^{L} \sum_{h=1}^{N} b_{k h}^{l}(t) H_{k h l}\left(x_{k}(t), x_{h}(t)\right)\right] \mathrm{d} t } \\
\mathrm{~d} x_{k}^{*}(t)= & {\left[(\tilde{\mu}-\tilde{\rho})\left(x_{k}^{*}(t)-\tilde{\mu} x_{k}(t)\right)-\xi_{k} x_{k}(t)+\sum_{l=1}^{L} \sum_{h=1}^{N} b_{k h}^{l}(t)\right.} \\
& \left.\times H_{k h l}\left(x_{k}^{*}(t), x_{h}^{*}(t)\right)\right] \mathrm{d} t, k \in \mathcal{N},
\end{aligned}\right.
$$

Then system (34) can be transformed into the following form

$$
\mathrm{d} X_{k}(t)=\left[\hat{F}_{k}\left(X_{k}(t), t\right)+\sum_{l=1}^{L} \sum_{k=1}^{N} b_{k h}^{l}(t) H_{k h l}\left(X_{k}(t), X_{h}(t)\right)\right] \mathrm{d} t
$$

where $X_{k}(t)=\left(x_{k}(t), x_{k}^{*}(t)\right)^{\mathrm{T}}, \quad \hat{F}_{k}\left(X_{k}, t\right)=$ $\left(\begin{array}{lllll}x_{k}^{*}(t) & - & \tilde{\mu} x_{k}(t),(\tilde{\mu}- & - & \tilde{\rho}\end{array}\right)\left(x_{k}^{*}(t)\right.$ $\left.\left.\tilde{\mu} x_{k}(t)\right)-\xi_{k} x_{k}(t)\right), \quad H_{k h l}\left(X_{k}(t), X_{h}(t)\right)=$ $\left(H_{k h l}\left(x_{k}(t), x_{h}(t)\right), H_{k h l}\left(x_{k}^{*}(t), x_{h}^{*}(t)\right)\right) . \quad$ Suppose that there are positive constants such that $\hat{\rho}_{k}^{l}, \hat{\sigma}_{k}^{l}$ $H_{k h l}\left(x_{k}, x_{h}\right) \leq \hat{\rho}_{k}^{l}\left|x_{k}\right|+\hat{\sigma}_{k}^{l}\left|x_{h}\right|$. We define (33) as the drive system, and the corresponding response system can be described as

$\mathrm{d} Y_{k}(t)=\left[\hat{F}_{k}\left(Y_{k}(t), t\right)+\sum_{l=1}^{L} \sum_{k=1}^{N} b_{k h}^{l}(t) H_{k h l}\left(Y_{k}(t), Y_{h}(t)\right)+U_{k}(t)\right] \mathrm{d} t$

in which $Y_{k}(t)=\left(y_{k}(t), y_{k}^{*}(t)\right)^{\mathrm{T}}, y_{k}^{*}(t)=\dot{y}_{k}(t)+\tilde{\mu} y_{k}(t) . U_{k}$ is defined in (3). Next, set $\tilde{E}_{k}(t)=Y_{k}(t)-X_{k}(t)$, thus we 
can obtain the error system

$$
\begin{aligned}
\mathrm{d} \tilde{E}_{k}(t)= & {\left[\hat{F}_{k}\left(Y_{k}(t), t\right)-\hat{F}_{k}\left(X_{k}(t), t\right)+\sum_{l=1}^{L} \sum_{k=1}^{N} b_{k h}^{l}(t)\right.} \\
& \left.H_{k h l}\left(\tilde{E}_{k}(t) \tilde{E}_{h}(t)\right)+U_{k}(t)\right] \mathrm{d} t, k \in \mathcal{N}
\end{aligned}
$$

in which $H_{k h l}\left(\tilde{E}_{k}(t), \tilde{E}_{h}(t)\right)=H_{k h l}\left(Y_{k}(t), Y_{h}(t)\right)^{-}$ $H_{k h l}\left(X_{k}(t), X_{h}(t)\right)$. Then, we can deduce that

$$
\begin{aligned}
& \tilde{E}_{k}^{T}(t)\left(\hat{F}_{k}\left(Y_{k}(t), t\right)-\hat{F}_{k}\left(X_{k}(t), t\right)\right) \\
= & e_{k}(t) e_{k}^{*}(t)-\tilde{\mu} e_{k}^{2}(t)-\tilde{\mu}(\tilde{\mu}-\tilde{\rho}) e_{k}^{2}(t)+(\tilde{\mu}-\tilde{\rho}) e_{k} e_{k}^{*}(t)-\xi_{k} e_{k}(t) \\
\leq & \frac{1}{2} e_{k}^{2}(t)+\frac{1}{2} e_{k}^{*^{2}}(t)+\tilde{\mu}(\tilde{\mu}-\tilde{\rho}+1) e_{k}^{2}(t)+\frac{1}{2}(\tilde{\mu}-\tilde{\rho})^{2} e_{k}^{2}(t) \\
& +\frac{1}{2}(\tilde{\mu}-\tilde{\rho})^{2} e_{k}^{*^{2}}(t)+\frac{1}{2} \xi_{k} e_{k}^{2}(t)+\frac{1}{2} \xi_{k} e_{k}^{*^{2}}(t) \\
\leq & \max \left\{\frac{1}{2}+\tilde{\mu}(\tilde{\mu}-\tilde{\rho}+1)+\frac{1}{2}(\tilde{\mu}-\tilde{\rho})^{2}\right. \\
& \left.+\frac{1}{2} \xi_{k}, \frac{1}{2}+\frac{1}{2}(\tilde{\mu}-\tilde{\rho})^{2}+\frac{1}{2} \xi_{k}\right\}\left|\tilde{E}_{k}(t)\right|^{2} \triangleq \tilde{M}\left|\tilde{E}_{k}(t)\right|^{2},
\end{aligned}
$$

and $\left|H_{k h l}\left(X_{k}(t), X_{h}(t)\right)\right| \leq \hat{\rho}_{k}^{l}\left|x_{k}\right|+\hat{\sigma}_{k}^{l}\left|x_{h}\right| \leq$ $\hat{\rho}_{k}^{l}\left|X_{k}\right|+\hat{\sigma}_{k}^{l}\left|X_{h}\right|$. It is obvious that Assumptions 1-2 are satisfied.

Next, we will try to verify the conditions in Theorem 1 . We than take diagraph $(\mathcal{K}, \mathbb{B})$ with 21 nodes into consideration (see Figure. 10). The nonzero elements of weight matrices $\left(b_{k h}^{l}\right)$ are defined as follows

- $b_{1,7}^{2}=b_{1,9}^{1}=b_{3,9}^{3}=b_{4,10}^{3}=b_{5,20}^{4}=b_{6,7}^{2}=b_{6,21}^{4}=$ $b_{8,1}^{4}=b_{8,3}^{3}=b_{9,1}^{1}=b_{9,4}^{3}=b_{10,1}^{4}=b_{10,5}^{3}=b_{16,21}^{1,21}=$ $b_{17,6}^{4}=b_{19,13}^{1}=b_{21,5}^{2}=0.001\left(0.4 e^{-t}+0.2\right) ; b_{2,8}^{3}=$ $b_{3,18}^{4}=b_{15,20}^{1}=0.001\left(0.2 e^{-t}+0.35\right)$;

- $b_{1,8}^{4}=b_{1,11}^{1}=b_{5,11}^{2}=b_{13,18}^{1}=0.001\left(0.5 e^{-t}+\right.$ $0.2) ; b_{1,10}^{4}=b_{12,17}^{1}=0.001\left(0.2 e^{-t}+0.25\right)$;

- $b_{7,1}^{4}=b_{11,6}^{2}=0.001\left(0.4 e^{-t}+0.6\right) ; b_{2,17}^{4}=b_{11,1}^{1}=$ $b_{14,19}^{1}=b_{18,12}^{1}=b_{19,3}^{2}=b_{20,14}^{1}=0.001\left(0.2 e^{-t}+0.1\right)$;

- $b_{4,19}^{2}=b_{18,2}^{4}=b_{20,4}^{4}=0.001\left(0.4 e^{-t}+0.25\right) ; b_{7,2}^{2}=$ $0.0001\left(0.5 e^{-t}+0.5\right)$

- $b_{15,20}^{1}=0.001\left(0.2 e^{-t}+0.35\right) ; b_{21,15}^{1}=0.001\left(0.2 e^{-t}+\right.$ $0.2)$;

Next, set several constants as $\tilde{\mu}=0.3$, $\tilde{\rho}=0.0012$ and functions $\hat{F}_{k}\left(x_{k}(t),(t)\right)=0.145 x_{k}$, $H_{k h 1}\left(X_{k}(t), X_{h}(t)\right)=H_{k h 2}\left(X_{k}(t), X_{h}(t)\right)=$ $H_{k h 3}\left(X_{k}(t), X_{h}(t)\right)=H_{k h 4}\left(X_{k}(t), X_{h}(t)\right)=X_{k}-X_{h}$. Besides, parameters of event-triggered functions are defined as $\alpha=0.1, \beta=0.199, \delta_{1}=1.8, \delta_{2}=4.9$, and the control gain is chosen as $a_{k_{1}}=0.2$ and $a_{k_{2}}=0.21$. Though calculation the conditions in Theorem 1 can be satisfied.

Then, trajectories of drive system (33) and response system (34) are simulated in Figure 11 and Figure 12, and meanwhile the control is given in Figure 17. The trajectories of measurement error and its corresponding triggering functions, as well as time instants are described in Figure 13 and Figure 14, respectively. Finally, Figure 15 and Figure 16 shows trajectories of error system (35) with and without control.

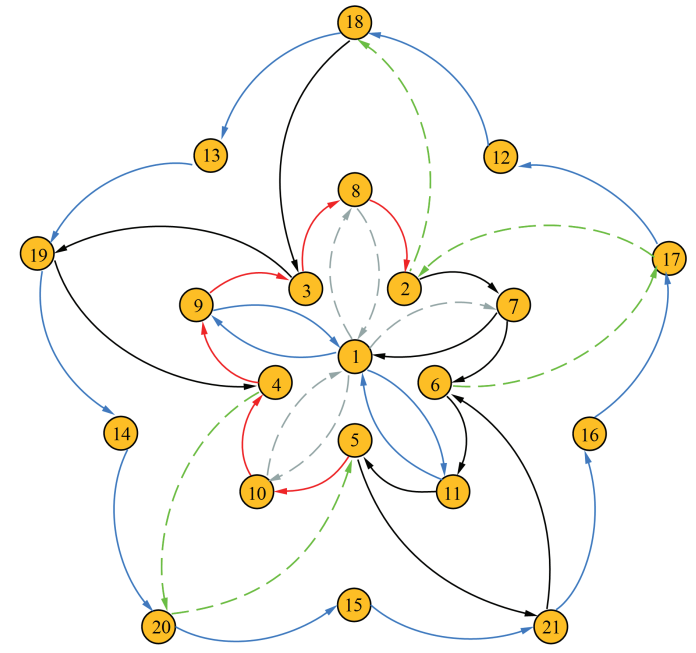

Fig. 10: A diagraph with 21 vertices

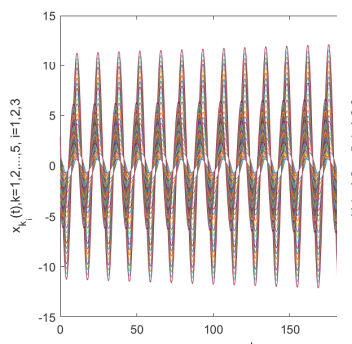

Fig. 11: Trajectories of drive system (33)

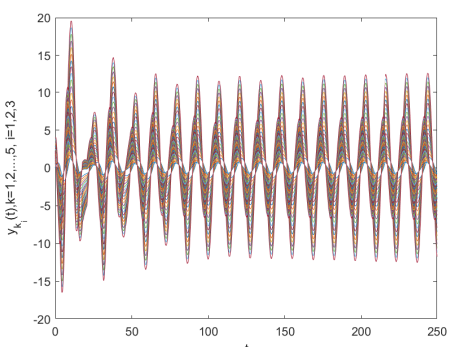

Fig. 12: Trajectories of response system (34)
Though the comparison, it is easy to find out that the drive and response systems are exponentially synchronized.

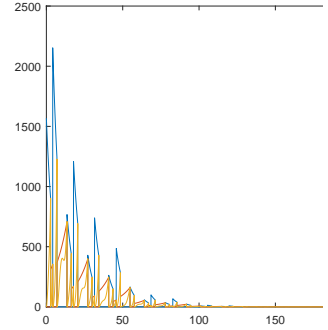

Fig. 13: Trajectories of triggering functions and measurement error

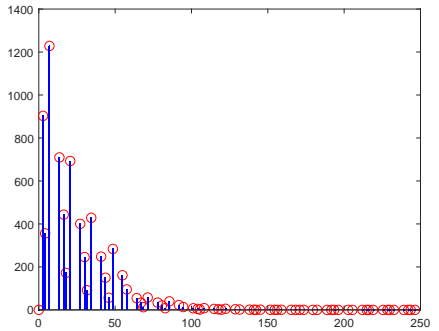

Fig. 14: The description of event-triggering time instants
Remark 6: Coupled oscillators have attracted many scholars' attention because they are widely applied in many fields [45]- [48]. In [45], periodically intermittent control is proposed to solve the synchronization problems of stochastic coupled oscillators with time-varying delays though graph-theoretical method. In [49], researchers studied the stability for networks of stochastic coupled systems with Lévy noise on the basis of graph-theoretic approach. In this paper, it is found out 


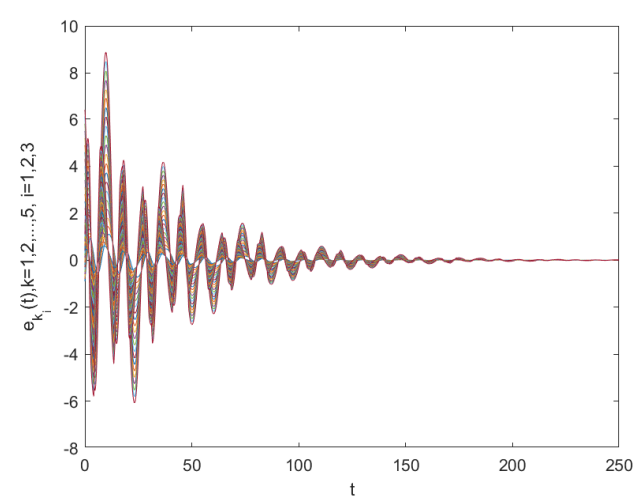

Fig. 15: Trajectories of error system (35) without control

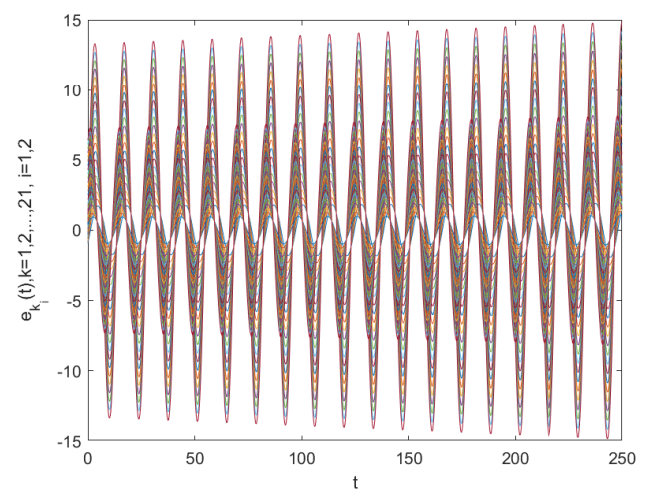

Fig. 16: Trajectories of error system (35) with control

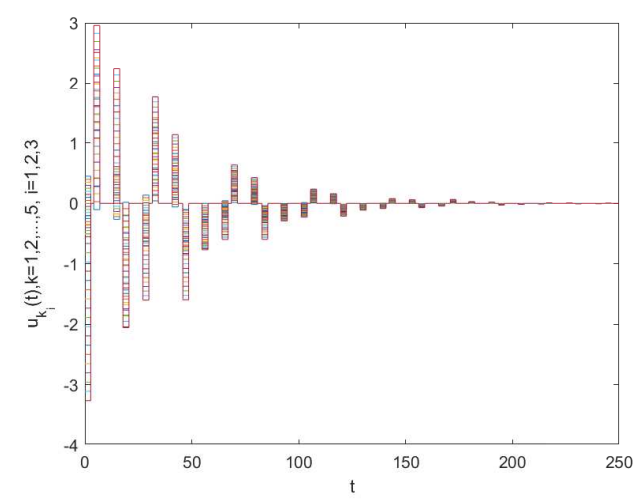

Fig. 17: Trajectories of control

that control gain $a_{k}$ as well as $\alpha$ and $\beta$ in the triggering functions plays an important role not only in convergence rate of exponential synchronization for system (33), but also in control widths and avoiding unnecessary information updates.

\section{Compliance with ethical standards}

Conflict of interest No potential conflict of interest was reported by the authors.

\section{REFERENCES}

[1] X. Yang, J. Lu. Finite-time synchronization of coupled networks with Markovian topology and impulsive effects. IEEE Trans. Autom. Control,
61(8): 2256-2261, 2016.

[2] H. Shen, J. Park, Z. Wu. Finite-time synchronization control for uncertain Markov jump neural networks with input constraints. Nonlinear Dyn., 77(4): 1709-1720, 2014.

[3] R. Manivannan, R. Samidurai, Q. Zhu. Further improved results on stability and dissipativity analysis of static impulsive neural networks with interval time-varying delays. J. Frankl. Inst. -Eng. Appl. Math., 354(14): 6312-6340, 2017.

[4] B. Boaretto, R. Budzinski, T. Prado, J. Kurths, S. Lopes. Neuron dynamics variability and anomalous phase synchronization of neural networks. Chaos, 28(10): 2018

[5] X. An, L. Zhang, Y. Li, J. Zhang. Synchronization analysis of complex networks with multi-weights and its application in public traffic network. Physica A, 412: 149-156, 2014.

[6] S. Deng, L. Huang, G. Xu, X. Wu, Z. Wu. On deep learning for trustaware recommendation in social networks. IEEE Trans. Neural Netw. Learn. Syst., 28(5): 1164-1177, 2017.

[7] J. Wang, Z. Qin, H. Wu, T. Huang, P. Wei. Analysis and pinning control for output synchronization and $\mathrm{H}$ infinity output synchronization of multiweighted complex networks. IEEE T. Cybern., 49(4): 1314-1326, 2019.

[8] H. Zhao, L. Li, H. Peng, M. Zheng. Impulsive control for synchronization and parameters identification of uncertain multi-links complex network. Nonlinear Dyn., 83(3): 1437-1451, 2016.

[9] J. Wang, P. Wei, H. Wu, T. Huang and M. Xu. Pinning synchronization of complex dynamical networks with multiweights. IEEE Trans. Syst. Man Cybern., 49(7): 1357-1370, 2019.

[10] C. Zhang, Y. Yang. Synchronization of stochastic multi-weighted complex networks with Levy noise based on graph theory. Physica A, 545: 2018.

[11] Y. Huang, J. Hou, E. Yang. General Decay Anti-Synchronization of Multi-Weighted Coupled Neural Networks with and without Reaction Diffusion Terms. Neural Comput. Appl., 32 (12): 8417-8430, 2020.

[12] M. Wang, R. Zheng, J. Feng, S. Qin, W. Li. Aperiodically intermittent control for exponential bipartite synchronization of delayed signed networks with multi-links. Chaos, 30(3): 2020.

[13] H. Zhang, T. Ma, G. Huang. Robust global exponential synchronization of uncertain chaotic delayed neural networks via dual-stage impulsive control. IEEE Trans. Syst. Man Cybern. Part B-Cybern., 40(3): 831-844, 2010.

[14] Z. Guan, Z. Liu, G. Feng, and Y. Wang. Synchronization of complex dynamical networks With time-Varying delays via impulsive distributed control. IEEE Trans. Circuits Syst. I-Regul. Pap., 57(8): 2182-2195, 2010.

[15] X. Li, S. Song. Stabilization of delay systems: delay-dependent impulsive control. IEEE Trans. Autom. Control, 62(1), 406-411, 2017.

[16] W. Yu, P. Delellis, G. Chen, M. Di Bernardo, J. Kurths. Distributed adaptive control of synchronization in complex networks. IEEE Trans. Autom. Control, 57(8): 2153-2158, 2012.

[17] Z. Cai, L.Huang. Finite-time stabilization of delayed memristive neural networks: discontinuous state-feedback and adaptive control approach. IEEE Trans. Neural Netw. Learn. Syst., 29(4) 856-868, 2019.

[18] B. Shen, Z. Wang, X. Liu. Sampled-data synchronization control of dynamical networks with stochastic sampling. IEEE Trans. Autom. Control, 57(10) 2644-2650, 2012.

[19] Y.Huang, H. Bao. Master-slave synchronization of complex-valued delayed chaotic Lur'e systems with sampled-data control. Appl. Math. Comput., 379: 2020.

[20] C. Hu, J. Yu, H. Jiang, Z. Teng. Exponential synchronization for reaction-diffusion networks with mixed delays in terms of p-norm via intermittent driving. Neural Netw., 31: 1-11, 2012.

[21] D. Zhang, Y. Shen, J. Mei. Finite-time synchronization of multi-layer nonlinear coupled complex networks via intermittent feedback control. Neurocomputing, 225: 129-138, 2017.

[22] C. Li, G. Feng, X. Liao. Stabilization of nonlinear systems via periodically intermittent control. IEEE Trans. Circuits Syst. II-Express Briefs, 54(11): 1019-1023, 2007.

[23] W. Xia, J. Cao. Pinning synchronization of delayed dynamical networks via periodically intermittent control. Chaos, 19(1): 821-91, 2009.

[24] X. Liu, T. Chen. Synchronization of nonlinear coupled networks via aperiodically intermittent pinning control. IEEE Trans. Neural Netw. Learn. Syst., 26(1): 113-126, 2015.

[25] X. Liu, T. Chen. Synchronization of complex networks via aperiodically intermittent pinning control. IEEE Trans. Autom. Control, 60(12) 33163321,2015 
[26] S. Li, Y. Li, X. Ding. More general results of aperiodically intermittent synchronization for stochastic Markovian switching complex networks with multi-links and time-varying coupling structure. Neurocomputing, 395: 39-55, 2020.

[27] D. Yue, Z. Guan, T. Li, R. Liao, F. Liu, L. Qiang. Event-based cluster synchronization of coupled genetic regulatory networks. Physica A, 482: 649-665, 2017.

[28] J. Sun, J. Liu, Y. Wang, Y. Yu, C. Sun. Fixed-time event-triggered synchronization of a multilayer Kuramoto-oscillator network. Neurocomputing, 379: 214-226, 2020.

[29] J. Lang, Y. Zhang, B. Zhang. Event-triggered network-based synchronization of delayed neural networks. Neurocomputing, 190: 155-164, 2016.

[30] H. Li, X. Liao, G. Chen, David. JH, Z. Dong, T. Huang. Event-triggered asynchronous intermittent communication strategy for synchronization in complex dynamical networks. Neural Netw., 66: 1-10, 2015.

[31] W. Zhu, Z. Jiang. Event-based leader-following consensus of multi-agent systems with input time delay. IEEE Trans. Autom. Control, 60(5): 13621367, 2015.

[32] X. Ni, Y. Cao, Z. Guo, T. Huang, S. Wen. Global exponential antisynchronization for delayed memristive neural networks via eventtriggering method. Neural Comput. Appl., 32(17) 13521-13535, 2020.

[33] T. Hu, X. Liu, Z. He, X. Zhang, S. Zhong. Hybrid event-triggered and impulsive control strategy for multiagent systems with switching topologies. IEEE T. Cybern., PP: 2020.

[34] X. Li, D. Peng, J. Cao. Lyapunov stability for impulsive systems via event-triggered impulsive control. IEEE Trans. Autom. Control, 65(11): 4908-4913, 2020.

[35] Z. Luo, W. Xiong, J. Cao, C. Huang. Event-triggered state tracking for two-dimensional neural networks with impulsive learning control schemes. J. Frankl. Inst.-Eng. Appl. Math., 357(17) 12364-12379, 2020.

[36] D. Peng, X. Li. Leader-following synchronization of complex dynamic networks via event-triggered impulsive control. Neurocomputing, 412: $1-10,2020$.

[37] D. West. Introduction to graph theory. Prentice hall Upper Saddle River, NJ, 2: 1996.

[38] M. Li, Z. Shuai. Global-stability for coupled systems of differential equations on networks. J. Differ. Equ., 248(1): 1-20, 2010.

[39] Y. Liu, W. Li, J. Feng. The stability of stochastic coupled systems with time-varying coupling and general topology structure. IEEE Trans. Neural Netw. Learn. Syst., 29(9): 4189-4200, 2018.

[40] M. Liu, H. Jiang, C. Hu. Finite-time synchronization of delayed dynamical networks via aperiodically intermittent control. Journal of the Franklin Institute, 354(13): 5374-5397, 2017.

[41] X. Tan. Event-based impulsive control for nonlinear systems and its application to synchronization of Chuas circuit. IMA J. Math. Control Inf., 37(1): 82-104, 2020.

[42] Y. Luo, J. Liu, D. Wei, L. Cao, R. Zhou, Y. Cao, X. Ding. A robust image encryption algorithm based on Chua's circuit and compressive sensing. Signal Process, 161: 227-247, 2019.

[43] S. Li, C. Lv, X. Ding. Synchronization of stochastic hybrid coupled systems with multi-weights and mixed delays via aperiodically adaptive intermittent control. Nonlinear Anal.-Hybrid Syst., 35: 2020.

[44] X. Wang, P. Miao. Finite-time function projective synchronization in complex multi-links networks and application to Chua's circuit. Int. J. Control Autom. Syst., 18(8): 1993-2001, 2020.

[45] B. Guo, Y. Wu, Y. Xiao, C. Zhang. Graph-theoretic approach to synchronizing stochastic coupled systems with time-varying delays on networks via periodically intermittent control. Appl. Math. Comput., 331: 341-357, 2018

[46] S. Li, H. Su, X. Ding. Synchronized stationary distribution of hybrid stochastic coupled systems with applications to coupled oscillators and a Chuas circuits network. Journal of the Franklin Institute, 355(17): 8743-8765, 2018

[47] S. Majhi, M. Perc, D. Ghosh. Chimera states in a multilayer network of coupled and uncoupled neurons. Chaos, 27(7): 2017.

[48] D. Deplano, M. Franceschelli, A. Giua, S.Luca. Distributed fiedler vector estimation with application to desynchronization of harmonic oscillator networks. IEEE Control Syst. Lett., 5(2): 659-664, 2021.

[49] C. Zhang, W. Li, K. Wang. Graph theory-based approach for stability analysis of stochastic coupled systems with Levy noise on networks. IEEE Trans. Neural Netw. Learn. Syst., 26(8): 1698-1709, 2019. 
Figures

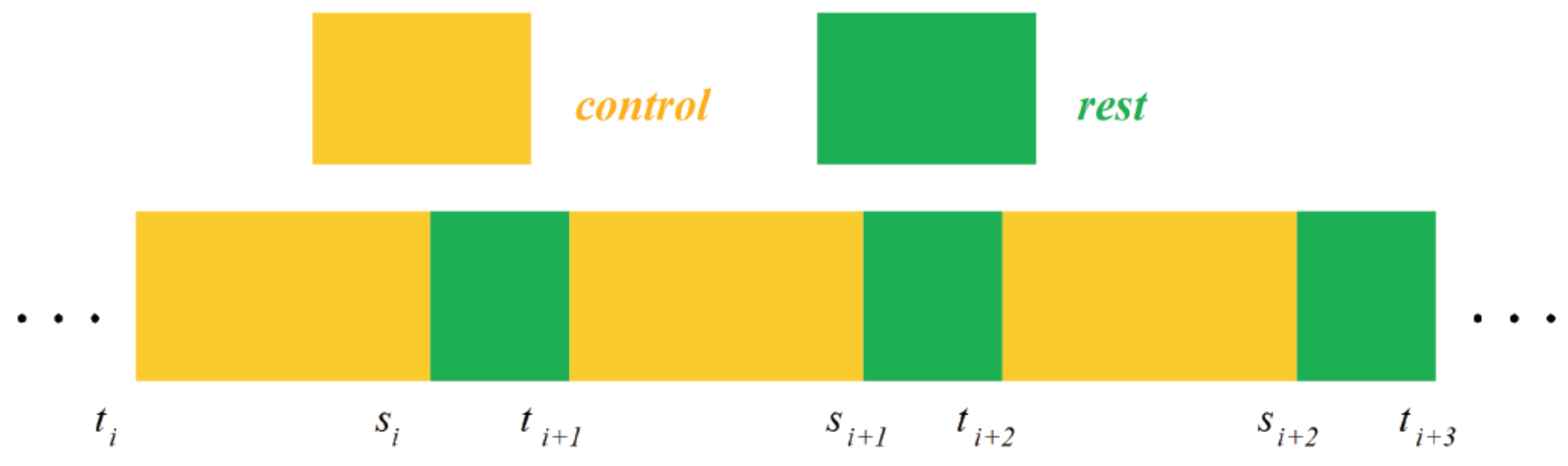

Figure 1

Sketch map of AIC strategy 


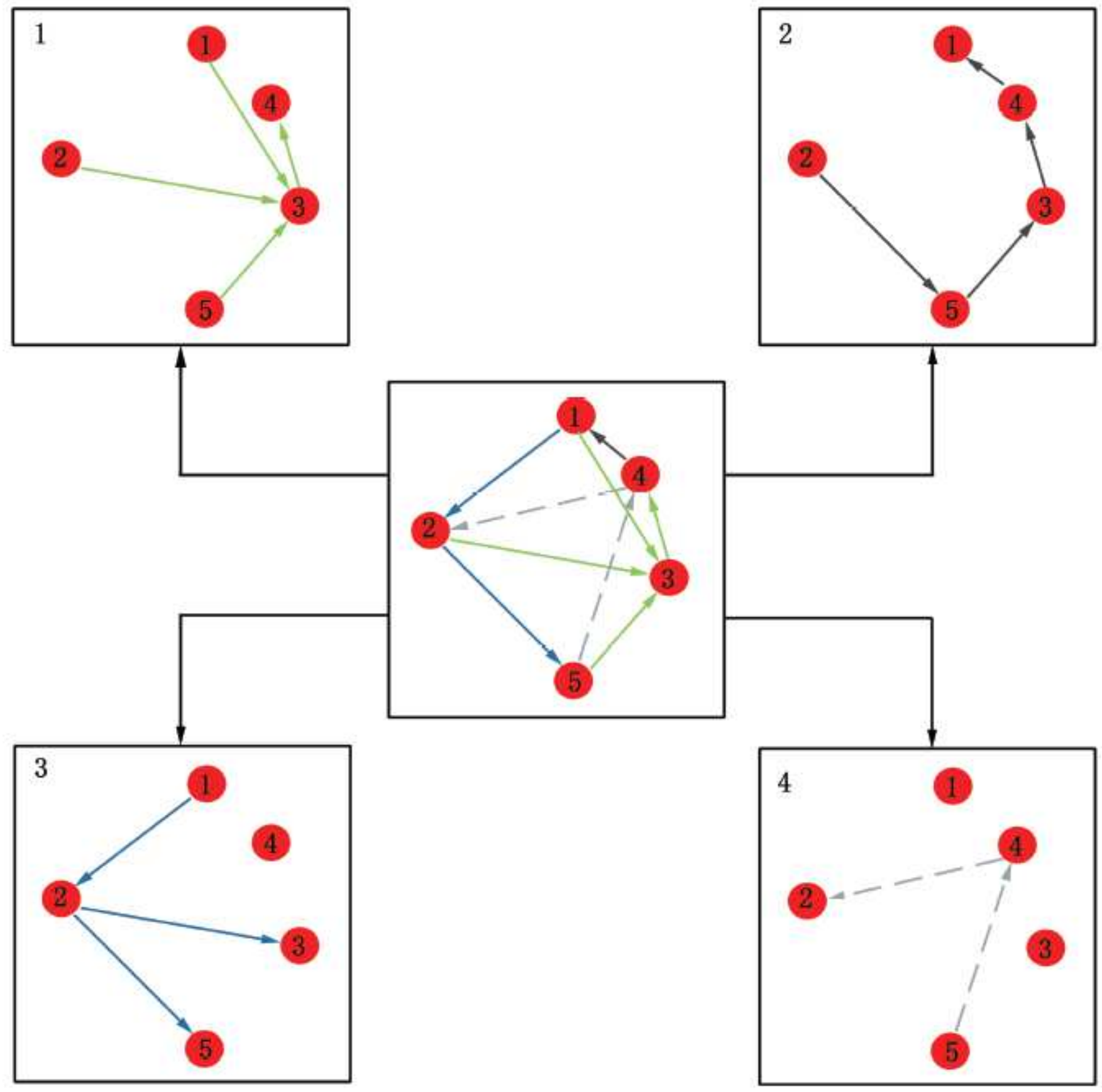

Figure 2

Digraph $(\mathrm{K}, \mathrm{B})$ and its four sub-digraphs $(\mathrm{K}, \mathrm{B} 1),(\mathrm{K}, \mathrm{B} 2),(\mathrm{K}, \mathrm{B} 3)$ and $(\mathrm{K}, \mathrm{B} 4)$ 


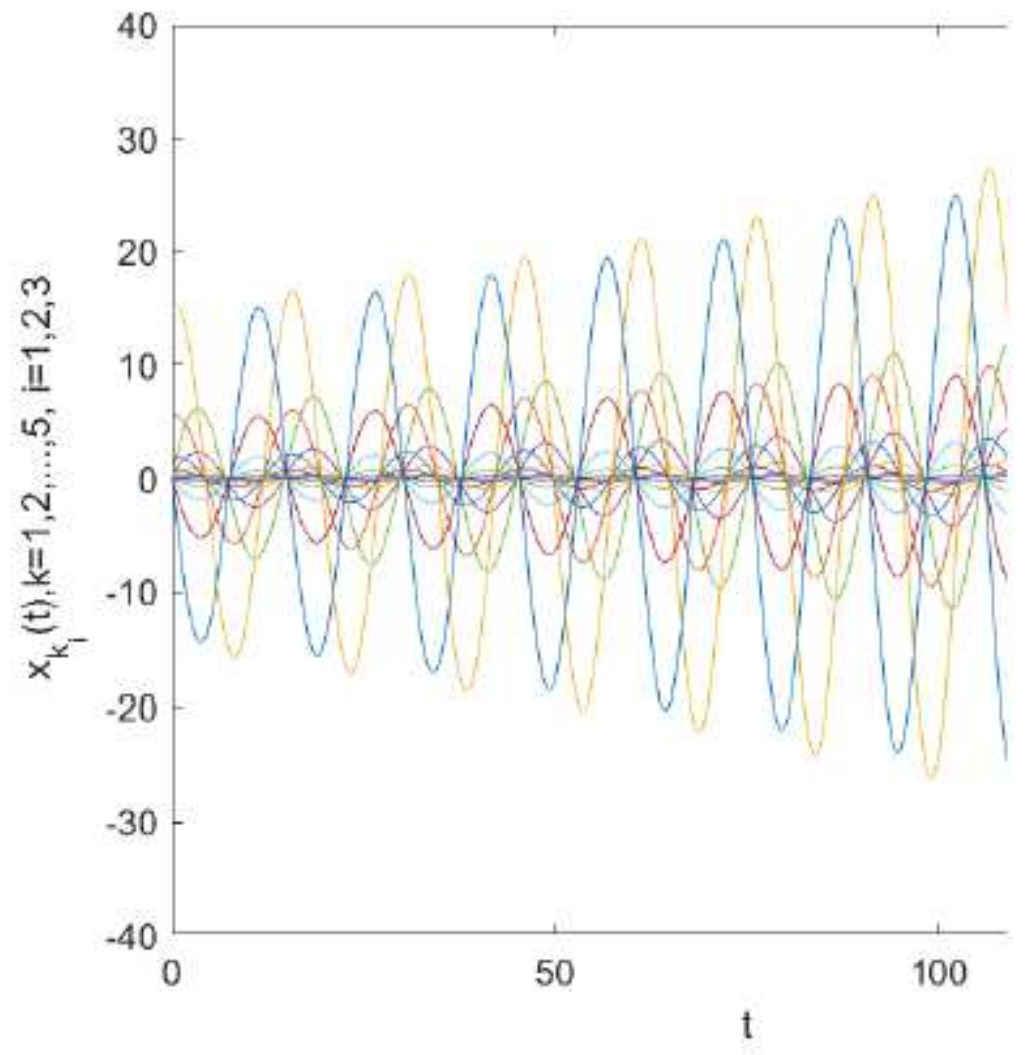

Figure 3

Trajectories of drive system (28)

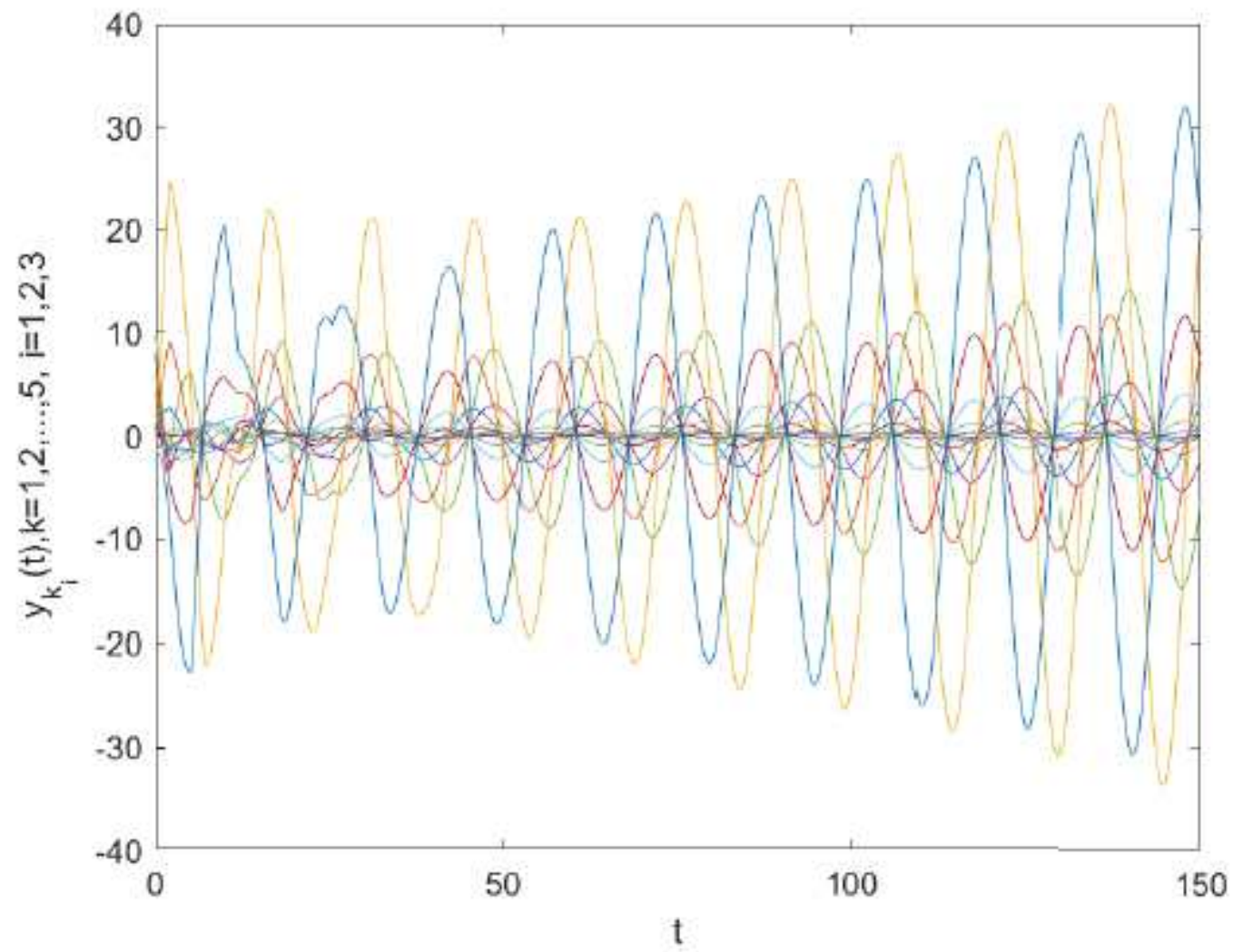

Figure 4 
Trajectories of response system (29)

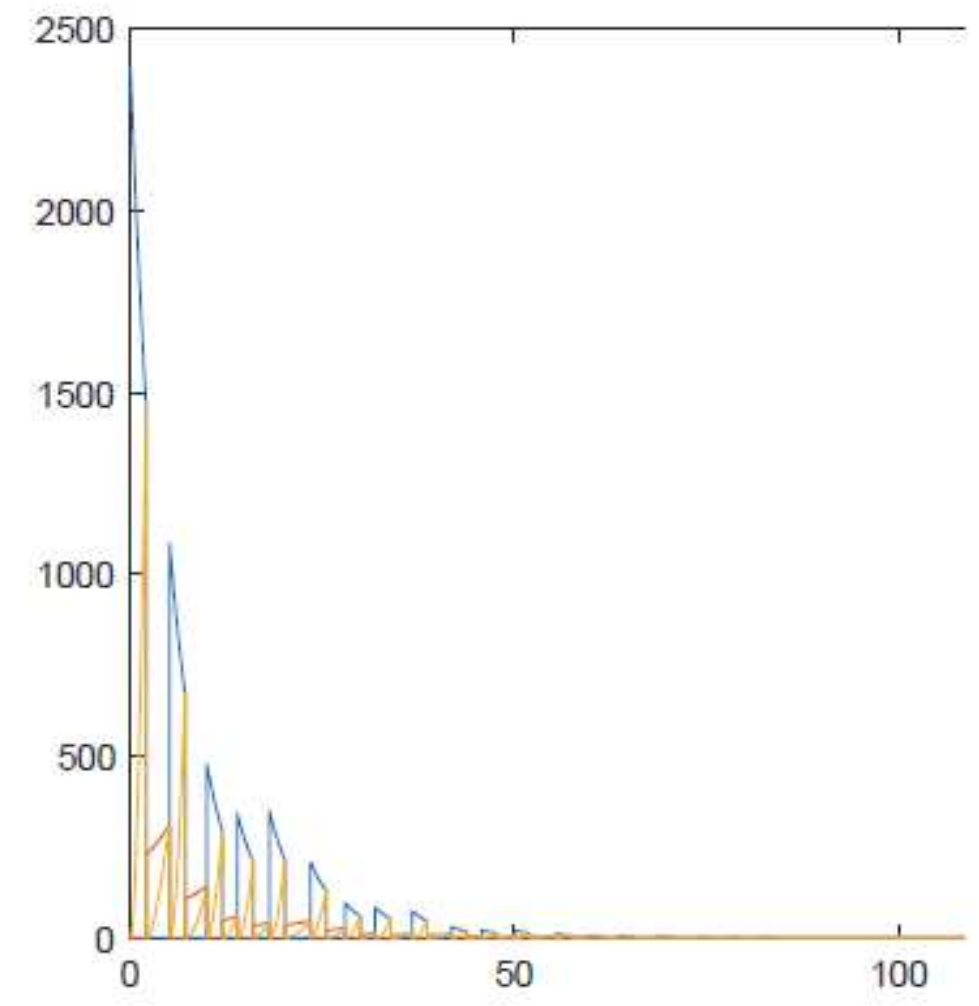

Figure 5

Trajectories of triggering functions and measurement error 


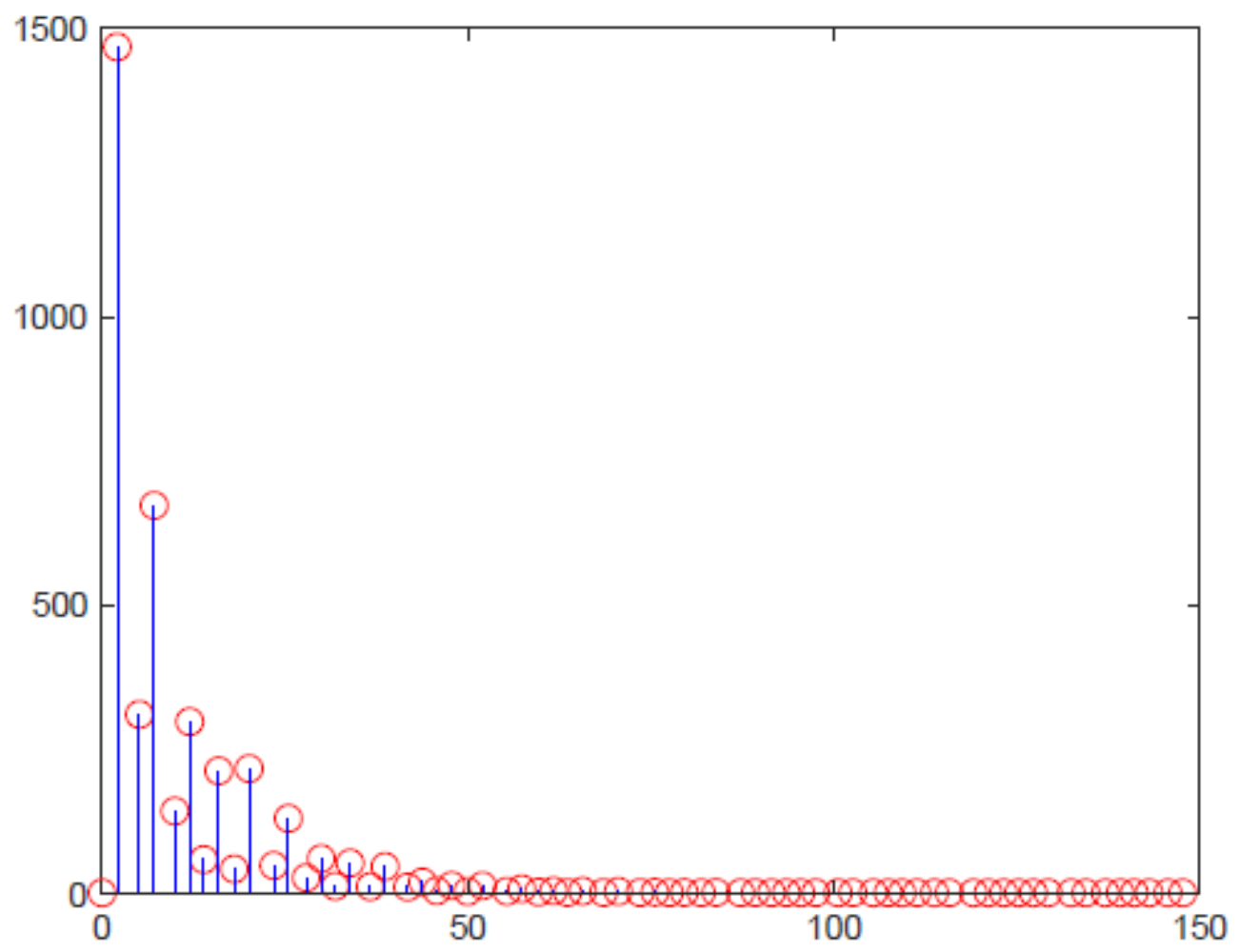

Figure 6

The description of event-triggered time instants 


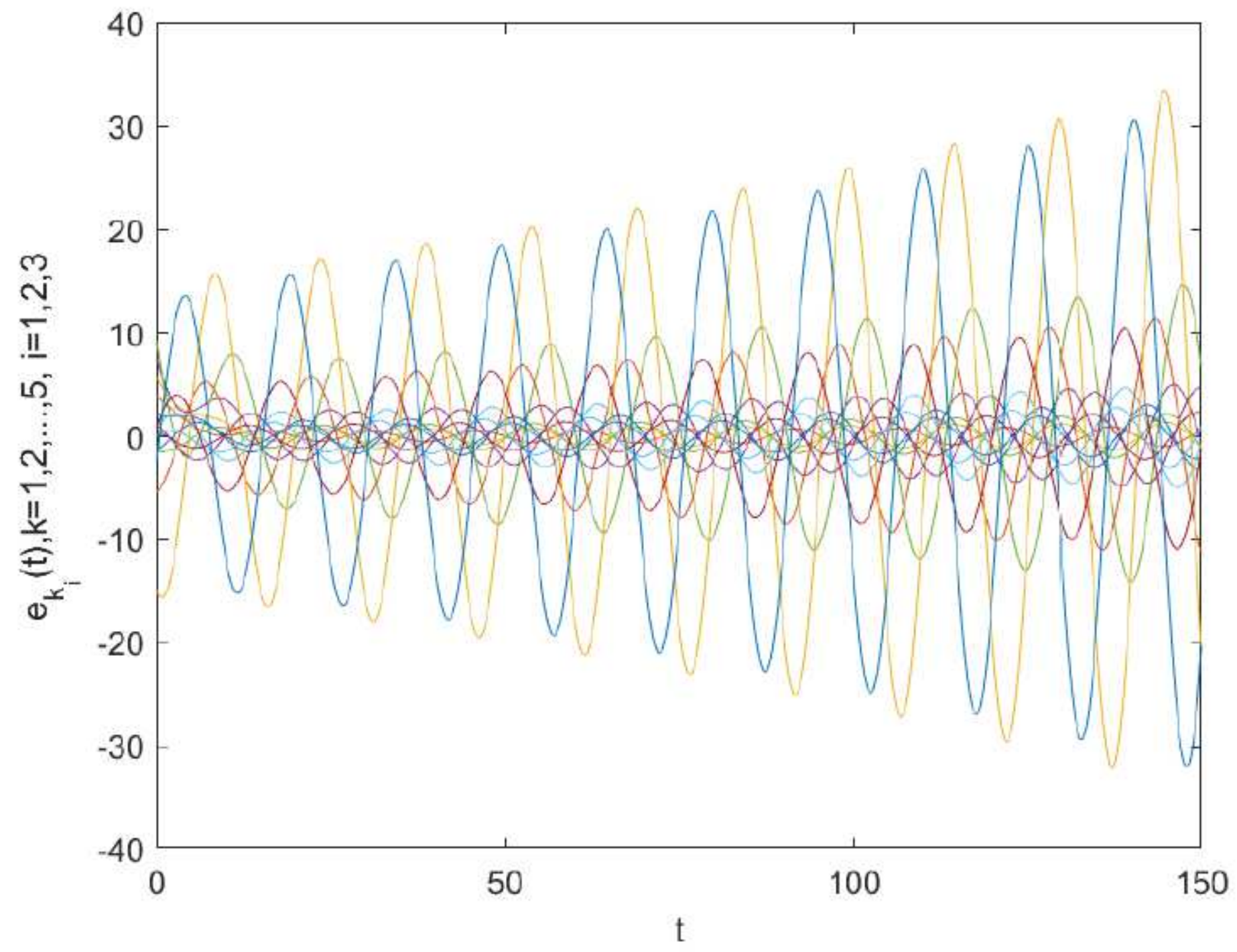

Figure 7

Trajectories of error system (30) without control 


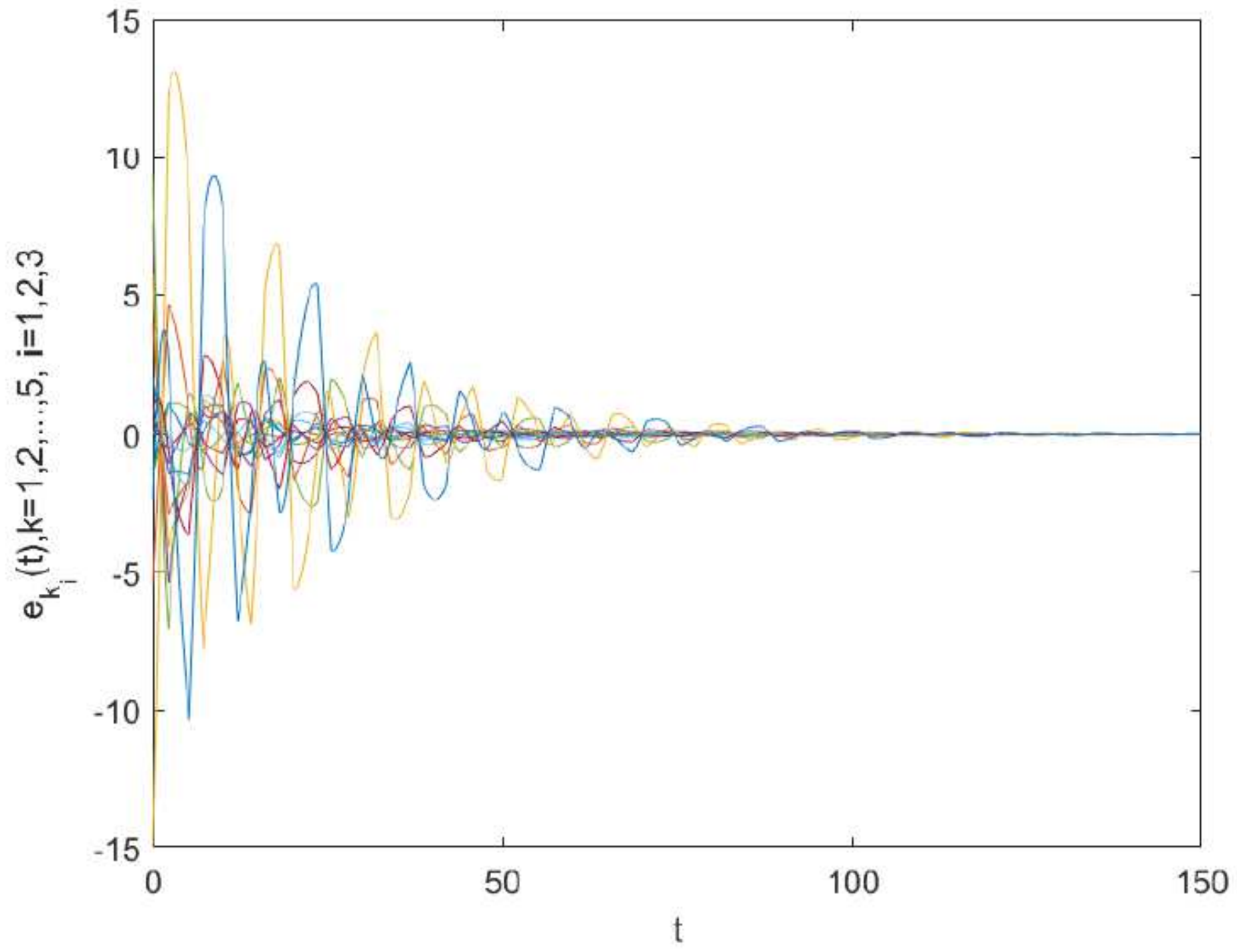

Figure 8

Trajectories of error system (30) with control 


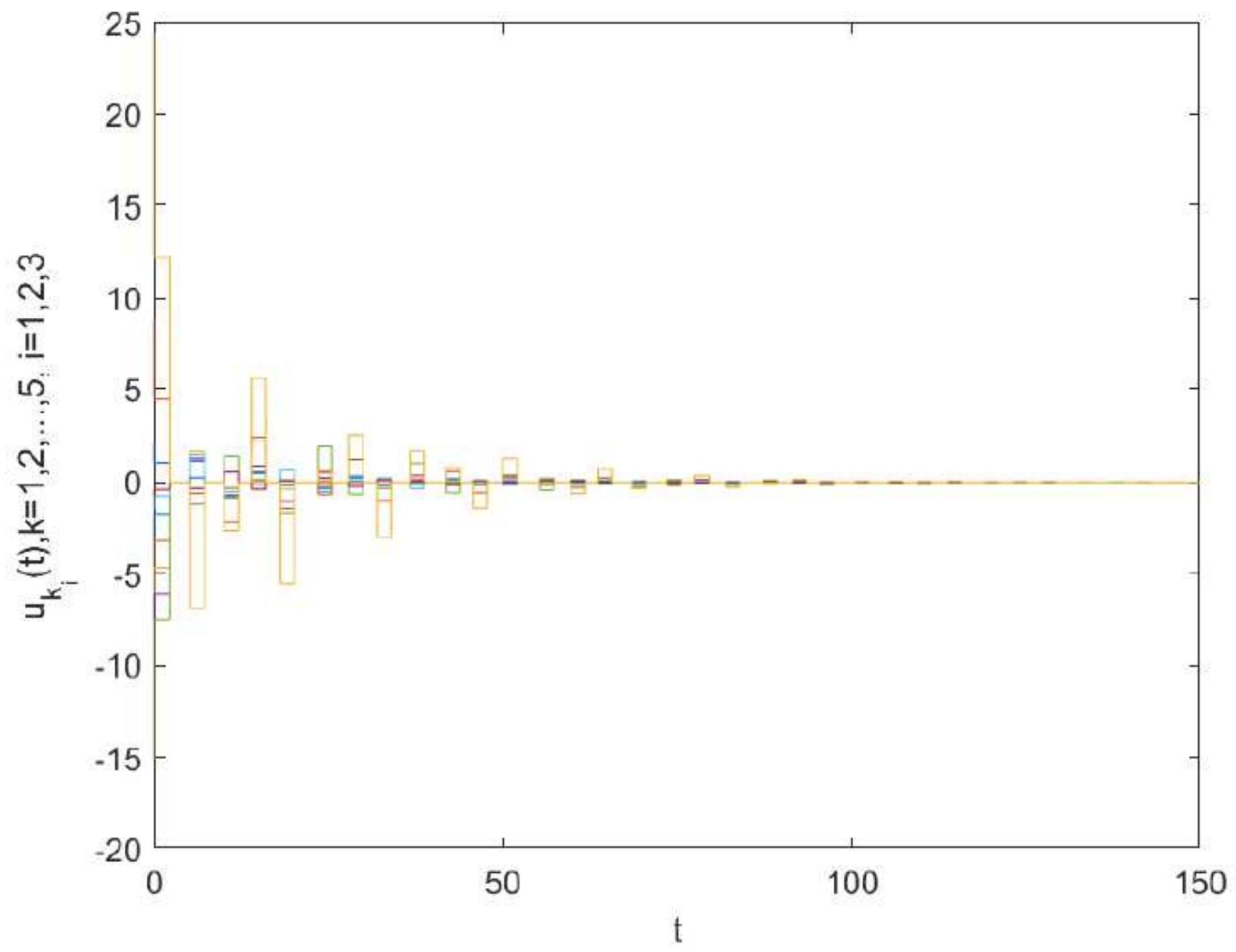

Figure 9

Trajectories of control 


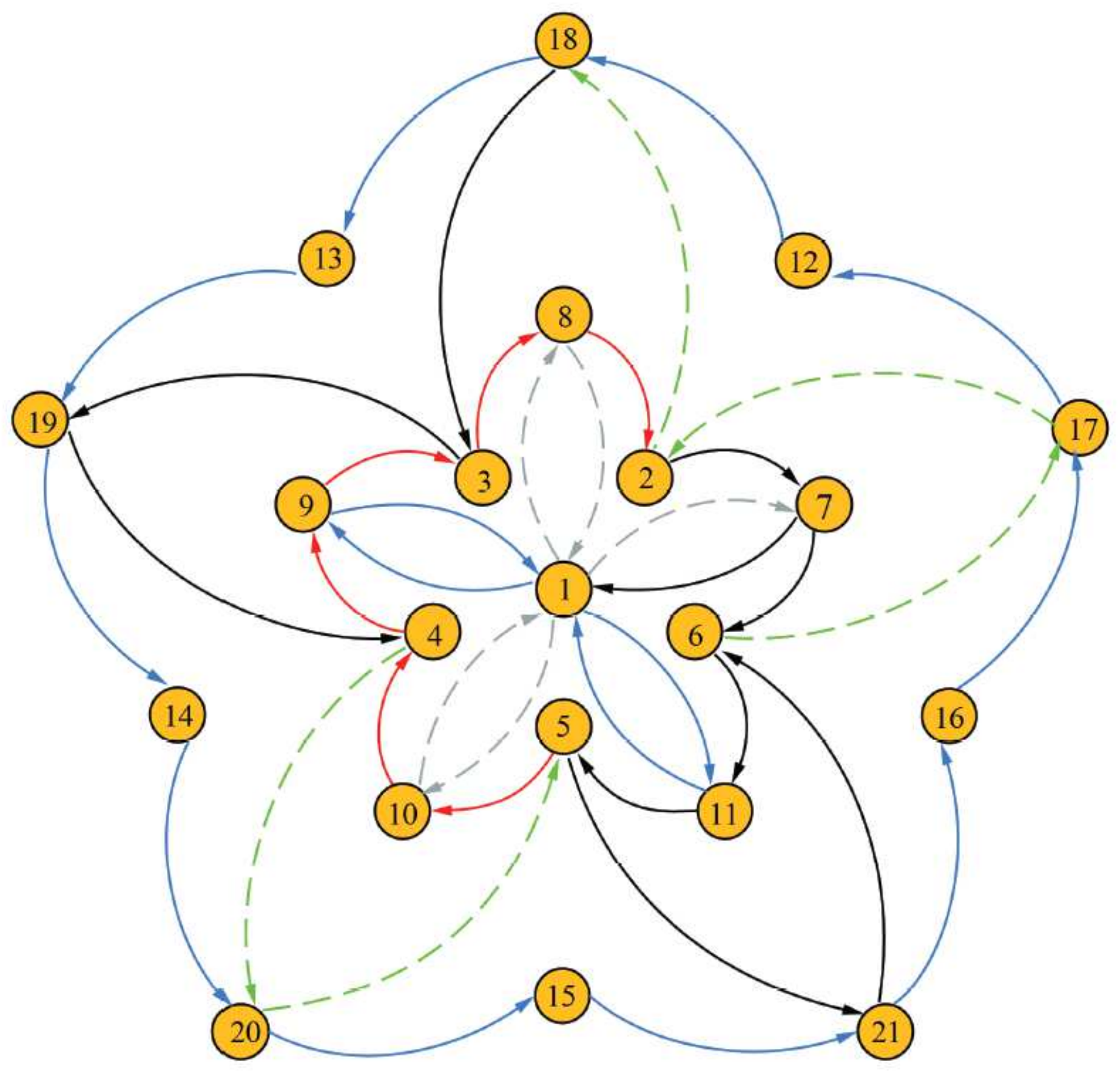

Figure 10

A diagraph with 21 vertices 


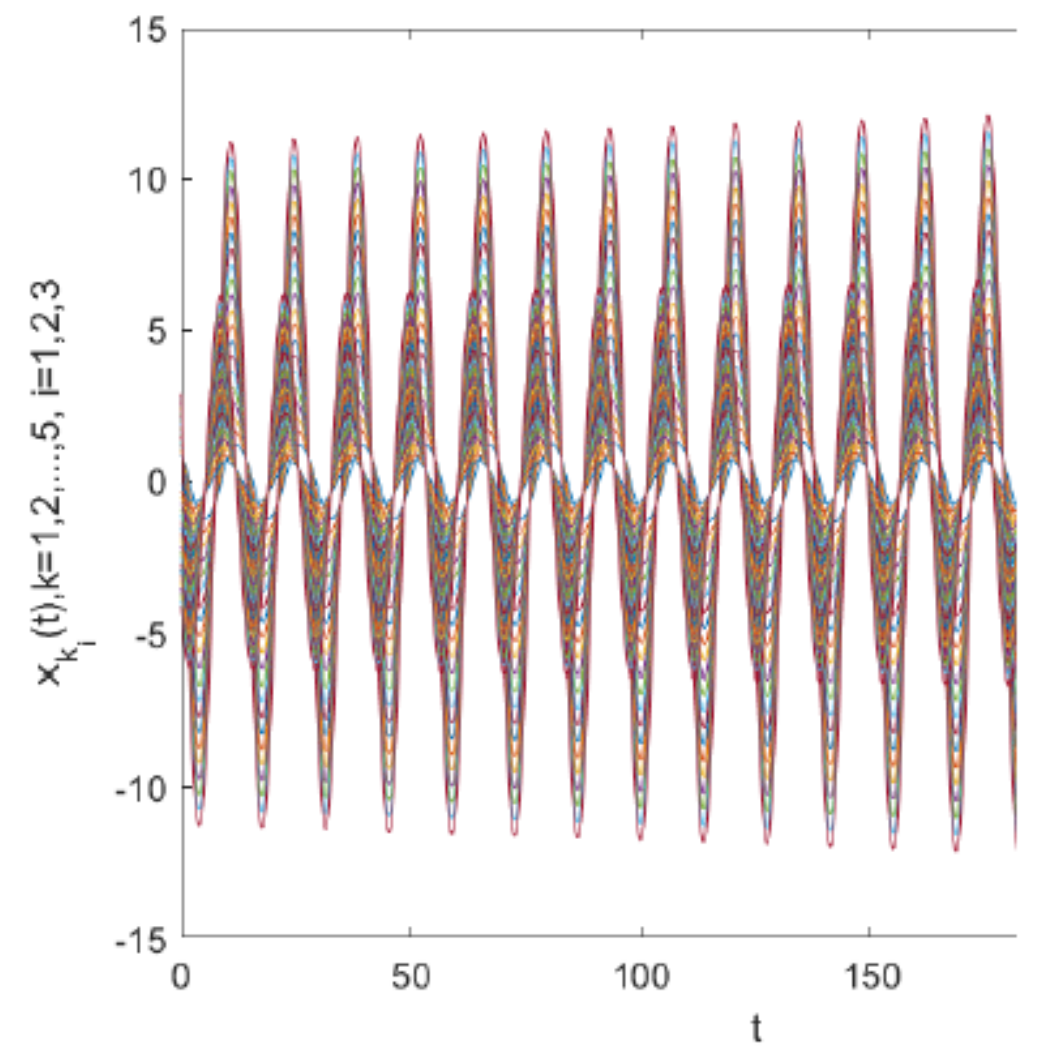

Figure 11

Trajectories of drive system (33)

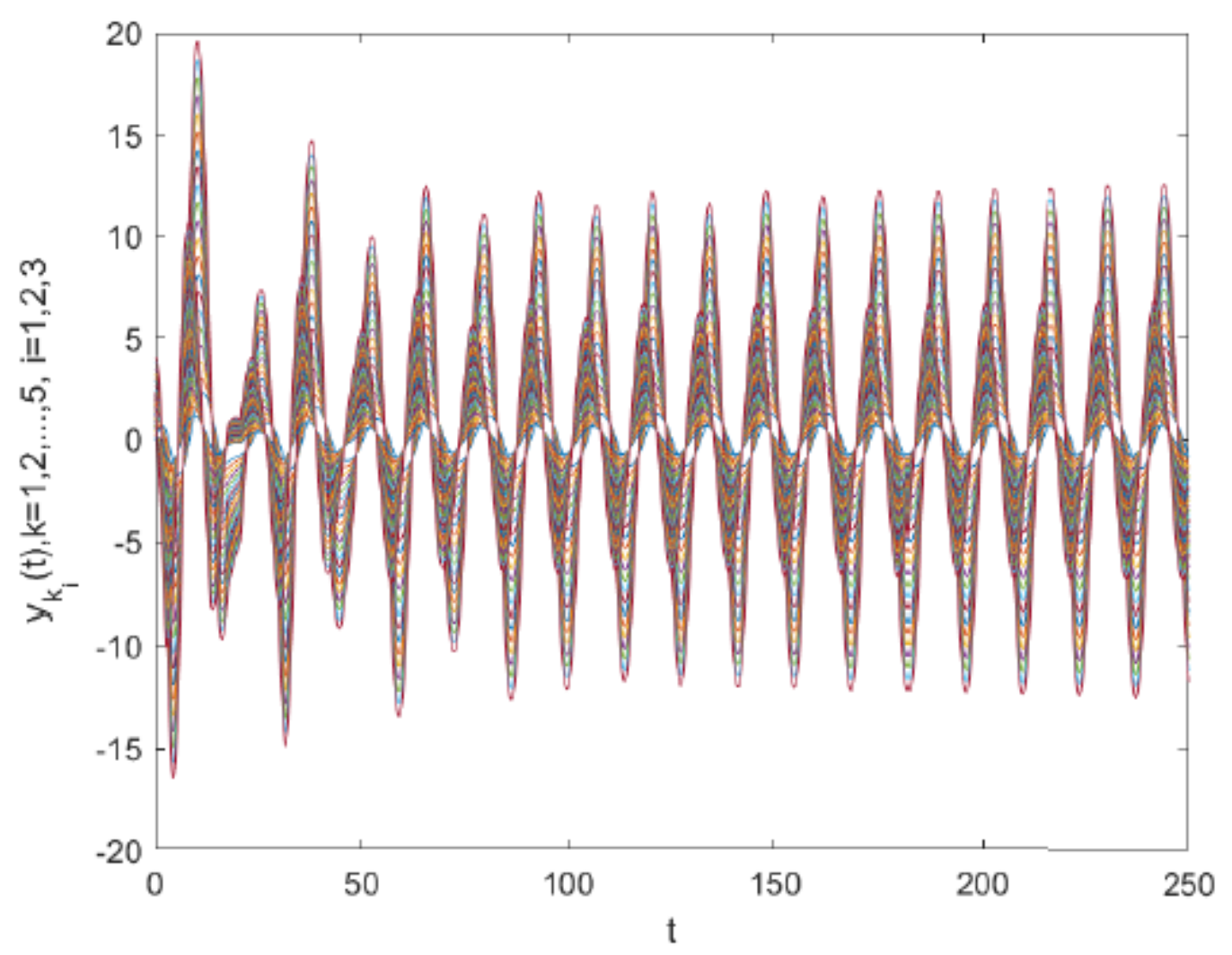


Figure 12

Trajectories of response system (34)

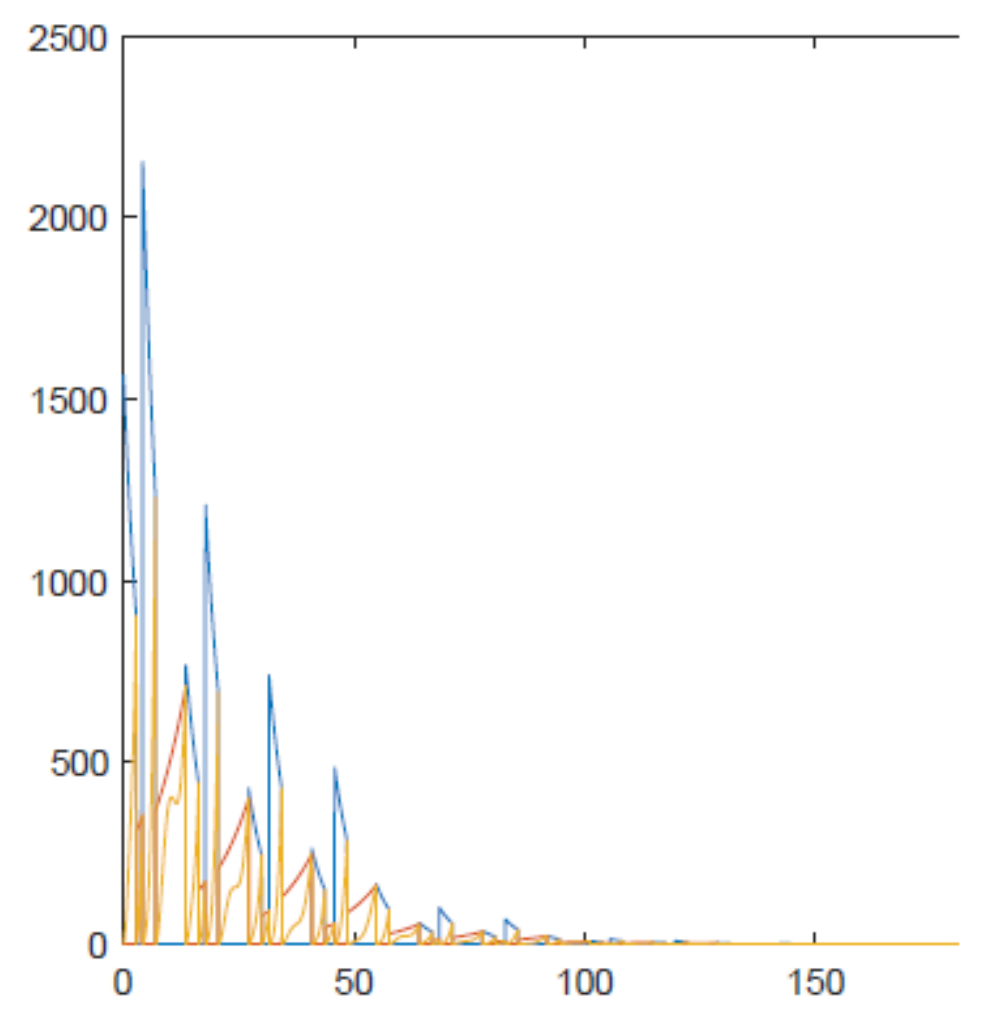

Figure 13

Trajectories of triggering functions and measurement error 


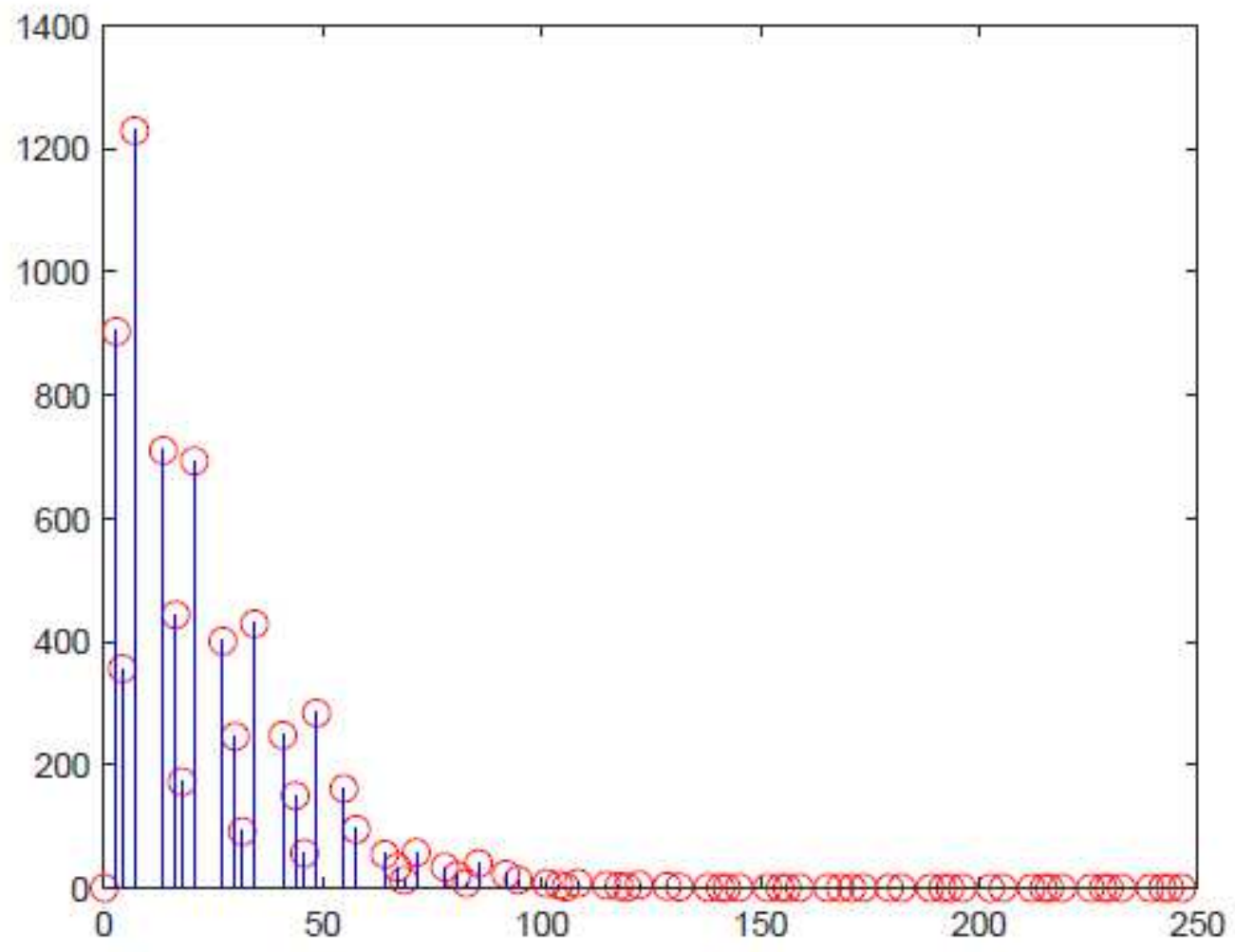

Figure 14

The description of event-triggering time instants 


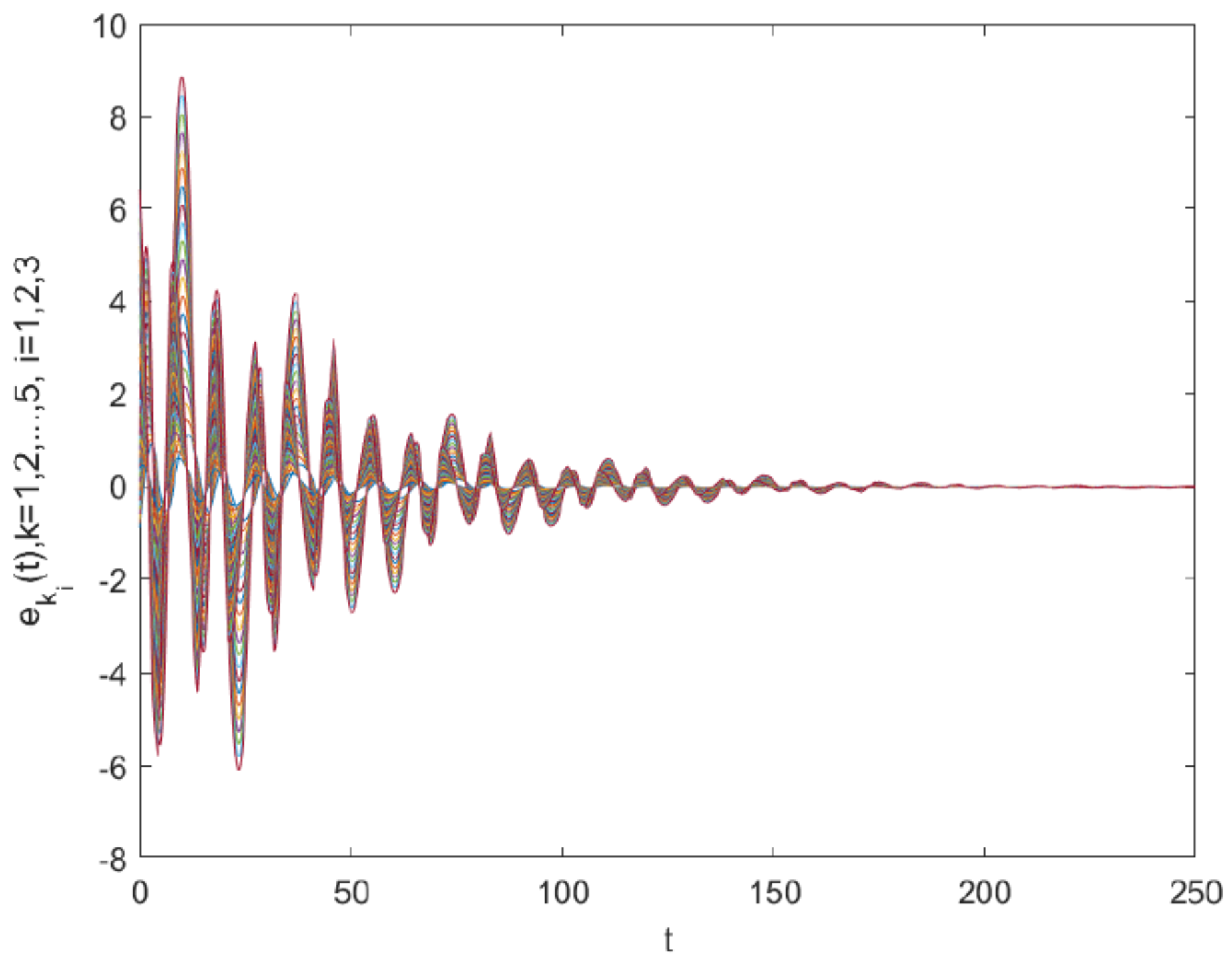

Figure 15

Trajectories of error system (35) without control 


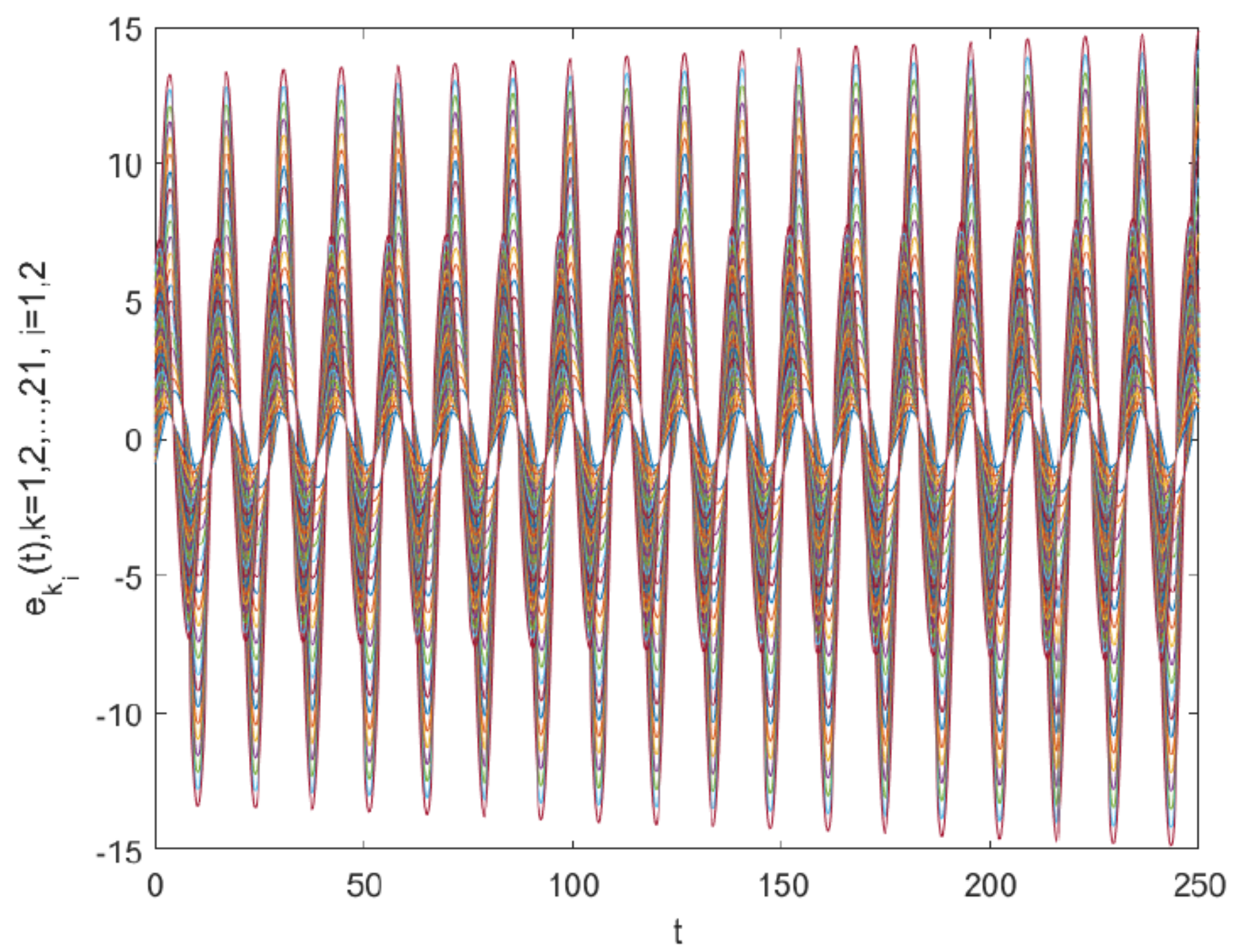

Figure 16

Trajectories of error system (35) with control 


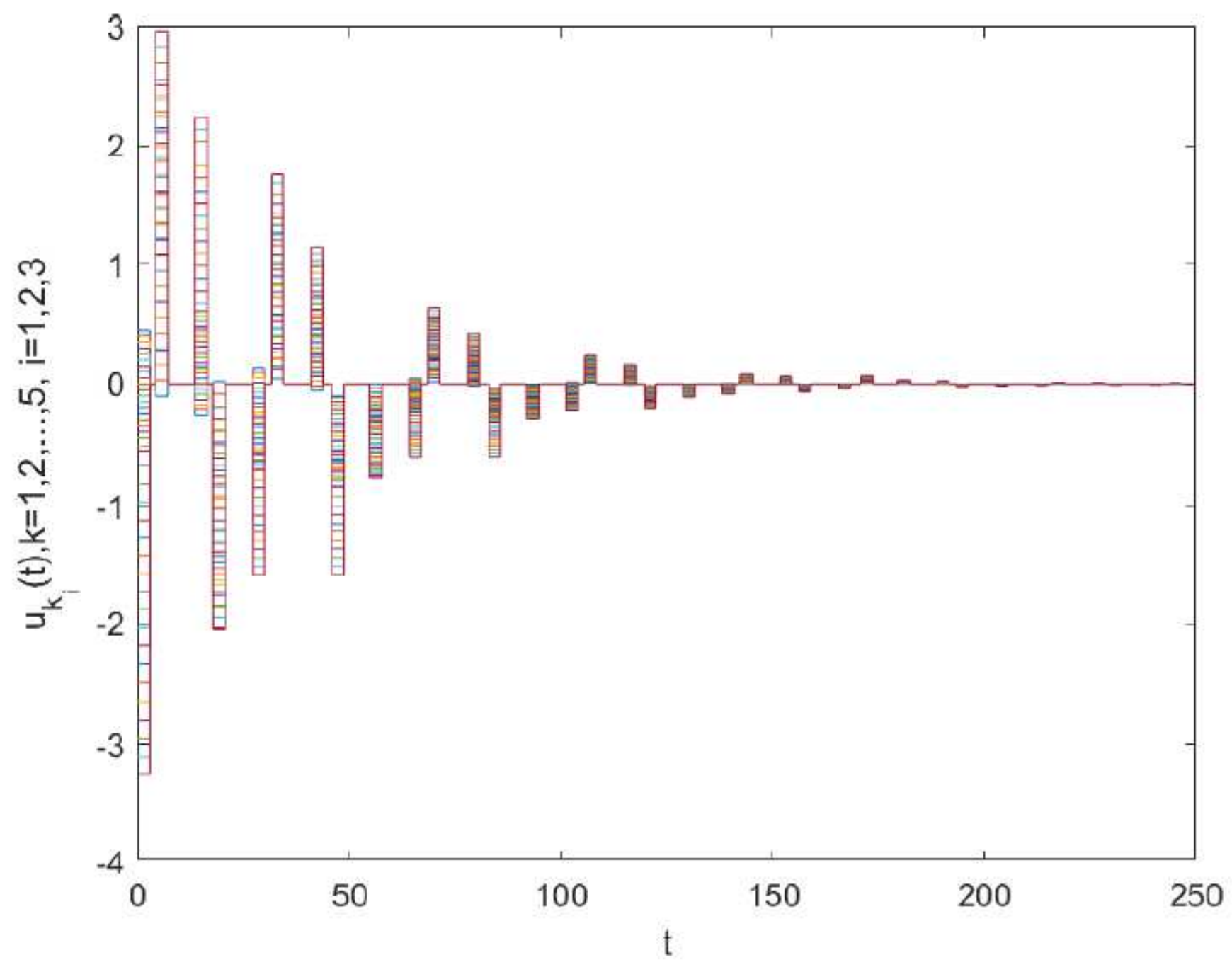

Figure 17

Trajectories of control 